\title{
Local bending stiffness identification of beams using simultaneous Fourier-series fitting and shearography
}

Filip Zastavnik, Rik Pintelon, Mathias Kersemans, Wim Van Paepegem, Lincy Pyl 2017-09-11

\begin{abstract}
In this paper, we present a novel method for the identification of the local bending stiffness of a beam. We use shearography to capture measurements of vibrating beams, so the input data for the identification is the modal slope - the differential of the modal shape. The modal slope is fitted by two Fourier-series functions, one of which is derived from a thin-beam model. The local bending stiffness is identified as the one corresponding with the best match between the measured and the two fitted modal slopes. This identification method, which we call simultaneous Fourier-series fitting, is demonstrated on numerically-generated inputs, as well as on experimental measurements. We use a flat, concave and convex beam, as well as beams with locally varying bending stiffness mimicking local damage to verify the method. It is shown that the method gives accurate results and is robust to noise. Additionally, it has advantageous properties that make it useful and practical: using this method, it is possible to perform the identification from only a sub-region of a beam and even without specifying the boundary conditions.
\end{abstract}

\section{Introduction}

From the ubiquitous usage of finite-element method (FEM) as a modelling tool in mechanical, civil and aeronautical engineering, the need emerged to accu- 
rately determine the stiffness, being one of the main input data in the model besides the geometry. Finding the stiffness in a database of materials will likely introduce some discrepancies as there will always be some differences between the real material and the one in the database. A better way to determine the stiffness is using a simple tensile test, though this also comes with drawbacks. For example, the real stiffness is never perfectly homogeneous, which is one of the basic assumptions of a tensile test.

Number of stiffness identification methods have been developed over the years. Overviews of these methods, with application examples mostly for identification of homogeneous stiffness, are discussed in review papers by Bonnet and Constantinescu [1] and Avril et al. [2].

Stiffness degradation is a widely used indicator to discern whether or not there is damage in a structure. Damage identification techniques using vibrational characteristics - in particular from a point of view of continuous structural health monitoring (SHM) - are reviewed in a series of widely known papers from Doebling, Farrar et al. [3-8]. For beams, the direct comparison of the modal curvature between the damaged and the undamaged beam can yield a good qualitative estimation of the damage extent, which was demonstrated by Pandey et al. [9]. Ndambi et al. [10] successfully identified the damage of reinforced concrete beams. Extending this, many methods to better quantify the damage on beams have been developed. Another example of a damage identification in beams is a paper by Huhtala and Bossuyt [11], which showed that using a very small number of measurement points and Bayesian inference, the damage can be successfully localised and quantified on a cantilever beam. A comparison between different damage identification techniques is done by Dessi and Camerlengo [12].

Degradation of stiffness is particularly important when considering structures made from composite materials, e.g. fibre-reinforced polymers (FRP), and damage from repeated loading. Papers from Degrieck and Van Paepegem [13] and Sevenois and Van Paepegem [14] review the determination of fatigue in fibre composites and show that accurately determining the stiffness degradation could play an important role in characterising fatigue in composites. One of the methods that can be used to determine the degradation of stiffness is the 
Ultrasonic Polar Scan (UPS) by Kersemans et al. [15].

Shearography is an interferometric full-field measurement and visualization technique which can be used for damage identification. It is already widely used to qualitatively visualise delaminations, such as in the study by Kalogiannakis et al. [16]. Another example of qualitative application is the comparison of infrared thermography to shearography, to visualise the heat-induced deformation in concrete bonded to FRP, performed by Lai et al. [17]. De Angelis et al. [18] presented a method for calculating the depth and size of holes drilled in composite plates using shearography. Once the position and the resonant frequency of a defect are found, an optimization procedure estimates the damage from the frequency and the modal slope. The method was successful in finding the size, but less so in finding the depth of the defect. Lopes et al. [19] demonstrated and compared two methods for extracting modal slopes from shearography measurements. They used spatial filtering and repeated differentiation to show that high-order differentiation can help with qualitative damage detection in plates. Further extension of that research lead to work by Mininni et al. [20], which employed shearography to experimentally determine the location and the magnitude of damage using a dimensionless curvature damage factor. The method was successful in determining the location and magnitude of damage on several examples. Finite-element method updating (FEMU) was used to further improve the results when damage was present in multiple locations.

The local stiffness identification aims at a slightly higher target than damage identification. In some sense, damage identification can be seen as a qualitative analogue of the quantitative local stiffness identification. In damage identification, some interpretation will still be needed to qualify what constitutes a damage and what not. Local stiffness identification is fully quantitative in the sense that it retrieves a physical property of the measured object. On the other hand, there are damage identification methods which parametrize the damage as a stiffness reduction factor.

For beams, one of the first stiffness identification approaches was proposed by Maeck and De Roeck [21] where the local bending stiffness was determined from successive differentiation of the measured modal shape of a concrete beam. Using the progressively increasing damage it was possible to locally track the 
evolution of the damage.

Goldfeld [22] developed a beam stiffness identification method based on smoothing and FEMU which had its efficiency evaluated on numerical examples. Subsequent development of this method was applied to frames [23]. An extension of the method was done by Goldfeld and Elias [24] and used a polynomial function to represent permissible damage distribution and evaluated the model on both numerical as well as experimental data.

Most of the stiffness identification methods in some way parametrise the measurement. Polynomial basis functions are commonly used as parametrisation. The particularity of the method presented here is that is uses Fourier-series to describe the modal fields, though it is not the only one to use this approach. Nguyen et al. [25] extended the virtual fields method (VFM) by parametrising the virtual fields by the Fourier-series basis functions, instead of by the commonly used polynomial. This increased the computation speed several times. A numerical example is given of the identification of the static deformation of heterogeneous elastic isotropic material with known boundary conditions. The reported error is about 5\% when noise is added. The authors report having problems with ringing, i.e. the Gibbs phenomenon, near the discontinuities in stiffness. The method is further expanded [26] in order to be able to automatically determine the boundary condition as a part of the identification procedure.

In this paper, we present a new method for bending stiffness identification in beams and we validate it on numerical and experimental examples. The method uses a simultaneous Fourier-series fitting of the modal slope, measured using shearography. The main advantage stemming from our approach is that the boundary conditions do not need to be explicitly prescribed. This significantly simplifies the experimental setup since any arbitrary boundary condition can be used. It is even possible to identify bending stiffness from a partially measured beam because the beam regions outside of the measurement area can be taken just as a special case of the arbitrary boundary conditions. Additionally, by using shearography the measurements can be performed quickly and accurately. First, in section 2, we present the theoretical basis and principle of the new method. A very important parameter for the method is the number of harmonics which we experimentally investigate and find the optimal value (in section 3). 
The method is then applied to numerically simulated examples (section 4) and to experimental results (section 5) to show that the method successfully identifies the bending stiffness variation. Conclusions and the directions for future work are given at the end of the paper.

\section{Framework for beam bending stiffness identi- fication}

\subsection{Thin-beam theory}

The bending stiffness identification is based on the thin-beam theory, also known as the Euler-Bernoulli beam theory. As this theory is one of the foundations of modern structural engineering, its description can be found in many textbooks, e.g. $[27,28]$. We start from the local equation of motion of a continuously vibrating thin beam, which defines an equilibrium for each point at a coordinate $x$ :

$$
\frac{\partial^{2}}{\partial x^{2}}\left(E(x) I(x) \frac{\partial^{2} w(x, t)}{\partial x^{2}}\right)+\rho A(x) \frac{\partial^{2} w(x, t)}{\partial t^{2}}=0
$$

where

- the length $l_{x}$ of the beam lies along the $x$ axis,

- $w(x, t)$ is the displacement in the $z$ direction (out-of-plane),

- $A(x)=l_{y} \times b(x)$ is the area of the cross-section, itself a product of the beam width ${ }^{1} l_{y}$ and the thickness $b(x)$,

- $\rho$ is the density,

- $E(x)$ is the Young's modulus,

- $I(x)$ is the second moment of area (for a rectangular cross-section $I(x)=$ $\left.b^{3}(x) l_{y} / 12\right)$ and

- $t$ is the time.

The displacement can be written as $w(x, t)=w(x) e^{i \omega t}$, i.e. a product of a spatially dependent function $w(x)$ - the modal shape - and a harmonic function with a circular frequency $\omega$. Using this, the previous time-dependent differential

\footnotetext{
${ }^{1}$ Here, the beam width $l_{y}$ is taken to be constant and the thickness $b(x)$ is variable with $x$ One or both of these can be variable with $x$, it does not meaningfully impact the identification procedure.
} 
equation can be transformed into a time-independent equation of a modal shape as

$$
\frac{\mathrm{d}^{2}}{\mathrm{~d} x^{2}}\left(E(x) I(x) \frac{\mathrm{d}^{2} w(x)}{\mathrm{d} x^{2}}\right)-\rho A(x) \omega^{2} w(x)=0
$$

This equation can be seen as an equation of a beam loaded statically with a distributed pressure $p(x)$

$$
p(x)=\rho A(x) \omega^{2} w(x)
$$

The pressure $p(x)$ is induced by the inertial forces, which in turn are proportional to the modal shape $w(x)$.

In the following text some conventions are followed. Most variables in the preceding equations are functions of the $x$ coordinate. Unless this needs to be particularly emphasized, the dependence on the $x$ coordinate is implicitly assumed, to make the equations more readable. The first and second differential of the modal shape $w$, with respect to $x$, are denoted as $w_{x}$ and $w_{x x}$. The product of the Young's modulus $E$ and the second moment of area $I$ is called the bending stiffness $E I$. Young's modulus $E$ and the bending stiffness $E I$ can be computed one from another if the local beam geometry is known, which it typically is.

Successive integration of (2) leads to

$$
\begin{aligned}
\frac{\mathrm{d}\left(E I w_{x x}\right)}{\mathrm{d} x} & =\int_{0}^{x} p \mathrm{~d} x+k_{Q}=-Q(x) \\
E I w_{x x} & =\int_{0}^{x}-Q \mathrm{~d} x+k_{M}=-M(x)
\end{aligned}
$$

where $Q$ is the section shear force and $M$ is the section moment, while $k_{Q}$ and $k_{M}$ are the integration constants.

The relevant modal fields - modal shape $w$, modal slope $w_{x}$, modal curvature $w_{x x}$, pressure $p$, section shear force $Q$ and section moment $M$ - are mutually dependent through the equations (1) to (5). Their circular dependency can be 
further illustrated as

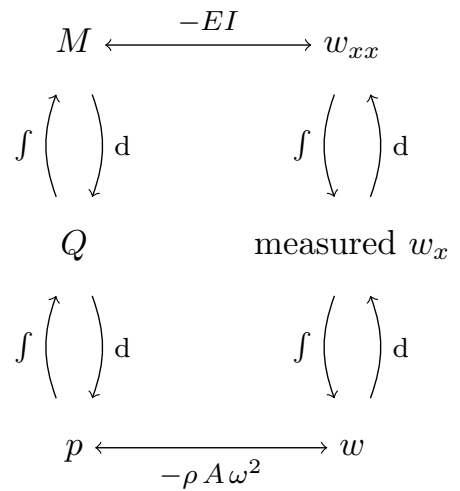

If any modal value is known over the whole beam, the other modal values can be reconstructed either through successive integration $\int$ or differentiation $\mathrm{d}$. Note that we did not have to prescribe boundary conditions at any point, since everything is derived from the local thin-beam equation.

\subsection{Fourier series as the basis function}

The Fourier series is chosen as the basis function to describe the modal fields. We represent some arbitrary 1-dimensional field $f$ by fitting Fourier-series function

$$
\tilde{f}(x, \boldsymbol{c})=c_{0}+\sum_{h=1}^{n_{h}}\left[c_{2 h-1} \cos (h \Omega x)+c_{2 h} \sin (h \Omega x)\right]
$$

where $n_{h}$ is the number of harmonics and $\Omega$ is the base frequency. It is convenient to represent the above equation in matrix form

$$
\tilde{\boldsymbol{f}}=\boldsymbol{F} \boldsymbol{c}
$$

where $\boldsymbol{c}=\left[c_{0}, \ldots, c_{2 n_{h}}\right]^{\top}$ is the coefficient vector. Field $\tilde{\boldsymbol{f}}$ is the fit to the measurement field $\boldsymbol{f}$ and $\boldsymbol{F}$ is the Fourier-series design matrix

$$
\boldsymbol{F}=\left[\begin{array}{cccccc}
1 & \cos \left(\Omega x_{1}\right) & \sin \left(\Omega x_{1}\right) & \cdots & \cos \left(n_{h} \Omega x_{1}\right) & \sin \left(n_{h} \Omega x_{1}\right) \\
\vdots & \vdots & \vdots & \vdots & \vdots & \vdots \\
1 & \cos \left(\Omega x_{n}\right) & \sin \left(\Omega x_{n}\right) & \cdots & \cos \left(n_{h} \Omega x_{n}\right) & \sin \left(n_{h} \Omega x_{n}\right)
\end{array}\right]
$$

Fitting the measured vector $\boldsymbol{f}$ by the function $\tilde{\boldsymbol{f}}$ means finding the coefficients $\boldsymbol{c}$. We can do this with a commonly-used technique, the linear least- 
squares method (LLSQ). Here we use the convention from programming language MATLAB and use a backslash operator "\" to represent the LLSQ [29]

$$
c=F \backslash f
$$

Important properties of the LLSQ fit are the residual and the cost function. When fitting by LLSQ we always find coefficients $c$ which minimize the cost function $K_{\text {fit }}$. The cost function is the sum of the squared residual

$$
K_{\mathrm{fit}}=\boldsymbol{R}_{\mathrm{fit}}^{\top} \boldsymbol{R}_{\mathrm{fit}}
$$

and the residual is the difference between the measured $\boldsymbol{f}$ and the fitted $\tilde{\boldsymbol{f}}$ data

$$
\boldsymbol{R}_{\mathrm{fit}}=\boldsymbol{f}-\tilde{\boldsymbol{f}}
$$

In case we want to associate weights with each measured point $i$, we can use the diagonal weighting matrix $\boldsymbol{V}$. The value of each element on the diagonal $(\boldsymbol{V})_{i, i}$ is usually related to the inverse of the variance of the measurement in that point (when multiple measurements are available). If we use the weighting matrix $\boldsymbol{V}$, we must also modify the cost function as

$$
K_{\mathrm{fit}}=\frac{1}{2} \boldsymbol{R}_{\mathrm{fit}}^{\top} \boldsymbol{V} \boldsymbol{R}_{\mathrm{fit}}
$$

and use weighted LLSQ to find the coefficients $c$

$$
\boldsymbol{c}=\left(\boldsymbol{V}^{\frac{1}{2}} \boldsymbol{F}\right) \backslash\left(\boldsymbol{V}^{\frac{1}{2}} \boldsymbol{w}_{x}\right)
$$

We will want to perform some operations on this fitted field. From the circle of relations between the modal fields in (6) we see that we will need some combination of differentiation, integration and multiplication.

The differential $\mathrm{d} \tilde{\boldsymbol{f}} / \mathrm{d} x$ can be calculated as

$$
\frac{\mathrm{d} \tilde{\boldsymbol{f}}}{\mathrm{d} x}=\frac{\mathrm{d}(\boldsymbol{F} \boldsymbol{c})}{\mathrm{d} x}=\frac{\mathrm{d} \boldsymbol{F}}{\mathrm{d} x} \boldsymbol{c}
$$

where

$\frac{\mathrm{d} \boldsymbol{F}}{\mathrm{d} x}=\left[\begin{array}{cccccc}0 & -\Omega \sin \left(\Omega x_{1}\right) & \Omega \cos \left(\Omega x_{1}\right) & \cdots & -n_{h} \Omega \sin \left(n_{h} \Omega x_{1}\right) & n_{h} \Omega \cos \left(n_{h} \Omega x_{1}\right) \\ \vdots & \vdots & \vdots & \vdots & \vdots & \vdots \\ 0 & -\Omega \sin \left(\Omega x_{n}\right) & \Omega \cos \left(\Omega x_{n}\right) & \cdots & n_{h} \Omega \sin \left(n_{h} \Omega x_{n}\right) & n_{h} \Omega \cos \left(n_{h} \Omega x_{n}\right)\end{array}\right]$ 
In the same manner, we can perform the differentiation further, to obtain second or higher differentials. Inversely, we can perform the integration.

Multiplication of two fields is performed element-wise by the Hadamard product, represented by "๑". For vectors, this is $(\boldsymbol{g} \odot \boldsymbol{f})_{i} \equiv(\boldsymbol{g})_{i} \cdot(\boldsymbol{f})_{i}$. We can alternatively perform multiplication as $\boldsymbol{g} \odot \boldsymbol{f}=(\boldsymbol{F} \odot \boldsymbol{g}) \boldsymbol{c}$ where a column $(\boldsymbol{F} \odot \boldsymbol{g})_{i} \equiv(\boldsymbol{F})_{i} \odot \boldsymbol{g}$. We represent element-wise power as $\left(\boldsymbol{f}^{\odot n}\right)_{i} \equiv(\boldsymbol{f})_{i}^{n}$.

The reason why we need these different ways of differentiating, integrating and multiplying the measured field is because in the identification method we want to be able to perform these operations on the design matrix instead of directly on the measurement.

\subsection{Identification principle}

The identification method exploits the fact highlighted in the circular relation of fields in (6) - by knowing any modal field, the others can be computed, either through successive integration or successive differentiation. In the matrix form, any modal field $\tilde{\boldsymbol{f}}$ is a product of its design matrix $\boldsymbol{F}$ and the coefficient vector $\boldsymbol{c}$. As we have seen in the previous section, successive differentiations, integrations and multiplications change only the design matrix while the coefficients $\boldsymbol{c}$ remain unchanged.

Starting point for the derivation of the fitting function equations can be any of the modal fields - the modal shape, modal slope, modal curvature, section moment or the section shear force. Since the modal slope $\boldsymbol{w}_{x}$ is the one which is measured, it is an obvious starting point. However, it is beneficial that the field that we fit is continuous in as many differentials as possible. While modal shape is surely continuous, the bending stiffness can be discontinuous. This can be an issue since the modal slopes are described by continuous functions. In a Fourier-series function this can result in a "ringing artefact" near the discontinuity location, an effect also known as the Gibbs phenomenon. Moment $M$ is sure to be at least $C^{2}$ (first and second derivative) continuous, no matter the distribution of the bending stiffness and the geometry of the beam, as the second derivative of $\boldsymbol{M}$, proportional to the modal shape $\boldsymbol{w}$, must be continuous. This makes the moment $\boldsymbol{M}$ the best candidate for fitting beams by the Fourier series. 
The moment field $\boldsymbol{M}$ is thus defined as

$$
M=F c
$$

From equations (4) and (5) we derive

$$
\frac{\mathrm{d}^{2} \boldsymbol{M}}{\mathrm{d} x^{2}}=-\boldsymbol{p}
$$

and from equation (3), we compute the modal slope $\tilde{\boldsymbol{w}}_{x}$

$$
\tilde{\boldsymbol{w}}_{x}=-\frac{\mathrm{d}}{\mathrm{d} x}\left(\frac{\mathrm{d}^{2} \boldsymbol{M}}{\mathrm{d} x^{2}} \odot\left(\rho \boldsymbol{A} \omega^{2}\right)^{\odot-1}\right)
$$

Second approach to obtain the modal slope (this second approach will bear the notation $\tilde{\tilde{\boldsymbol{w}}}_{x}$ and the associated design matrix will be $\tilde{\tilde{\boldsymbol{F}}}$ ) is by integrating the moment $\boldsymbol{M}$ from equation (5).

$$
\tilde{\tilde{\boldsymbol{w}}}_{x}=-\int_{0}^{x} \boldsymbol{M} \odot(\boldsymbol{E} \boldsymbol{I})^{\odot-1} d x+k_{M}
$$

where the $k_{M}$ is the integration constant. $k_{M}$ can be seen as yet another coefficient in the coefficient vector so that $\boldsymbol{c}=\left[c_{0}, \ldots, c_{2 n_{h}}, k_{M}\right]^{\top}$. For the design matrices $(\boldsymbol{F}$ and $\tilde{\boldsymbol{F}})$ which do not use this coefficient, the appropriate column is filled with zeros. Both $\tilde{\boldsymbol{w}}_{x}$ and $\tilde{\tilde{\boldsymbol{w}}}_{x}$ are two separate analytic functions that fit the measured modal slope $\boldsymbol{w}_{x}$.

Using modified design matrices

$$
\tilde{\boldsymbol{F}}=-\frac{\mathrm{d}}{\mathrm{d} x}\left(\frac{\mathrm{d}^{2} \boldsymbol{F}}{\mathrm{d} x^{2}} \odot\left(\rho \boldsymbol{A} \omega^{2}\right)^{\odot-1}\right)
$$

and

$$
\tilde{\tilde{\boldsymbol{F}}}=\int_{0}^{x} \boldsymbol{F} \odot(\boldsymbol{E} \boldsymbol{I})^{\odot-1} d x
$$

we can write equations (17) and (18) as

$$
\begin{aligned}
& \boldsymbol{w}_{x} \simeq \tilde{\boldsymbol{w}}_{x}=\tilde{\boldsymbol{F}} \boldsymbol{c} \\
& \boldsymbol{w}_{x} \simeq \tilde{\tilde{\boldsymbol{w}}}_{x}=\tilde{\tilde{\boldsymbol{F}}} \boldsymbol{c}
\end{aligned}
$$

The design matrices $\tilde{\boldsymbol{F}}$ and $\tilde{\tilde{\boldsymbol{F}}}$ are fully defined by components (sines and cosines) of the Fourier series and the physical properties of the beam - density $\rho$, crosssection $\boldsymbol{A}$, circular resonant frequency $\boldsymbol{\omega}$ and the distribution of the bending 
stiffness $\boldsymbol{E} \boldsymbol{I}$. From the physical properties, all but the bending stiffness distribution $\boldsymbol{E I}$ can be accurately measured and they are needed as the input properties for the bending stiffness identification method. Since the measurement grid is dense, the design matrices $\tilde{\boldsymbol{F}}$ and $\tilde{\tilde{\boldsymbol{F}}}$ can be accurately computed from $\boldsymbol{F}$ numerically, as described in the previous section.

Equations (21) and (22) are part of a simultaneous system of equations as they are defined by the same coefficient vector $c$

$$
\left[\begin{array}{c}
\boldsymbol{w}_{x} \\
\boldsymbol{w}_{x}
\end{array}\right] \simeq\left[\begin{array}{c}
\tilde{\boldsymbol{w}}_{x} \\
\tilde{\tilde{\boldsymbol{w}}}_{x}
\end{array}\right]=\left[\begin{array}{c}
\tilde{\boldsymbol{F}} \\
\tilde{\tilde{\boldsymbol{F}}}
\end{array}\right] \boldsymbol{c}
$$

which can be solved as a LLSQ fit

$$
\boldsymbol{c}=\left[\begin{array}{c}
\tilde{\boldsymbol{F}} \\
\tilde{\tilde{\boldsymbol{F}}}
\end{array}\right] \backslash\left[\begin{array}{c}
\boldsymbol{w}_{x} \\
\boldsymbol{w}_{x}
\end{array}\right]
$$

Further on, we will call this identification the simultaneous Fourier-series fitting as it fits a single measurement $\boldsymbol{w}_{x}$ by two design matrices simultaneously.

The cost function of the simultaneous Fourier-series fitting identification is

$$
K_{\mathrm{ID}}=\left[\begin{array}{c}
\tilde{\boldsymbol{R}}_{I D} \\
\tilde{\tilde{\boldsymbol{R}}}_{I D}
\end{array}\right]^{\top}\left[\begin{array}{c}
\tilde{\boldsymbol{R}}_{I D} \\
\tilde{\tilde{\boldsymbol{R}}}_{I D}
\end{array}\right]
$$

where the residuals are

$$
\begin{aligned}
\tilde{\boldsymbol{R}}_{I D} & =\tilde{\boldsymbol{w}}_{x}-\boldsymbol{w}_{x} \\
\tilde{\tilde{\boldsymbol{R}}}_{I D} & =\tilde{\tilde{\boldsymbol{w}}}_{x}-\boldsymbol{w}_{x}
\end{aligned}
$$

In case of the weighted LLSQ fitting, the cost function changes to

$$
K_{\mathrm{ID}}=\frac{1}{2}\left[\begin{array}{c}
\tilde{\boldsymbol{R}}_{I D} \\
\tilde{\tilde{\boldsymbol{R}}}_{I D}
\end{array}\right]^{\top}\left[\begin{array}{cc}
\boldsymbol{V} & \mathbf{0} \\
\mathbf{0} & \boldsymbol{V}
\end{array}\right]\left[\begin{array}{c}
\tilde{\boldsymbol{R}}_{I D} \\
\tilde{\tilde{\boldsymbol{R}}}_{I D}
\end{array}\right]
$$

The weighted fitting is then performed as

$$
\boldsymbol{c}=\left[\begin{array}{cc}
\boldsymbol{V}^{\frac{1}{2}} & \tilde{\boldsymbol{F}} \\
\boldsymbol{V}^{\frac{1}{2}} & \tilde{\tilde{\boldsymbol{F}}}
\end{array}\right] \backslash\left[\begin{array}{ll}
\boldsymbol{V}^{\frac{1}{2}} & \boldsymbol{w}_{x} \\
\boldsymbol{V}^{\frac{1}{2}} & \boldsymbol{w}_{x}
\end{array}\right]
$$


To actually identify the bending stiffness distribution $\boldsymbol{E I}$, the identification starts from an initial guess. The bending stiffness distribution is defined as a vector of $n_{\mathrm{ID}}$ identification control points distributed along the length of the beam, $\boldsymbol{E} \boldsymbol{I}_{\mathrm{ID}} \in \mathbb{R}^{n_{\mathrm{ID}} \times 1}$. The values in between the control points are interpolated by cubic splines. Finding the correct bending stiffness distribution $\boldsymbol{E I}$ is a nonlinear least squares optimization problem. The parameters of the optimization are the bending stiffness distribution $\boldsymbol{E} \boldsymbol{I}$ and the coefficients $\boldsymbol{c}$. Finding the coefficients $\boldsymbol{c}$ is, as shown before, a linear problem which can be solved efficiently by the LLSQ. The non-linear optimization is thus separated into the non-linear part, which iteratively adjusts only the $\boldsymbol{E I}$, and the linear part. For a given $\boldsymbol{E I}$, the corresponding optimal coefficients $\boldsymbol{c}$ and the cost function $K$ are found noniteratively (and thus very computationally efficiently) by the LLSQ method. In this way the non-linear optimization finds the optimal (the correct) $\boldsymbol{E} \boldsymbol{I}$ in a computationally efficient way.

Furthermore, measurements $\boldsymbol{w}_{x}^{(i)}$ of multiple modes, where "(i)" in the superscript signifies $i$-th mode at resonant frequency $f_{i}$, can be used simultaneously for the identification. We can define residuals $\tilde{\boldsymbol{R}}_{I D}^{(i)}$ and $\tilde{\tilde{\boldsymbol{R}}}_{I D}^{(i)}$ for a measurement $w_{x}^{(i)}$ and expand the cost function to include $n_{\text {meas }}$ of multiple measurements as

$$
K_{\mathrm{ID}}=\left[\begin{array}{c}
\tilde{\boldsymbol{R}}_{I D}^{(1)} \\
\tilde{\boldsymbol{R}}_{I D}^{(1)} \\
\vdots \\
\tilde{\boldsymbol{R}}_{I D}^{\left(n_{\text {meas }}\right)} \\
\tilde{\tilde{\boldsymbol{R}}}_{I D}^{\left(n_{\text {meas }}\right)}
\end{array}\right]^{\top}\left[\begin{array}{c}
\tilde{\boldsymbol{R}}_{I D}^{(1)} \\
\tilde{\boldsymbol{R}}_{I D}^{(1)} \\
\vdots \\
\tilde{\boldsymbol{R}}_{I D}^{\left(n_{\text {meas }}\right)} \\
\tilde{\boldsymbol{R}}_{I D}^{\left(n_{\text {meas }}\right)}
\end{array}\right]
$$

Notice that no information about the boundary condition is explicitly used in this identification. Though it is possible to modify the identification method to explicitly prescribe the boundary condition, this can be more of a drawback than a benefit. With the exception of the free boundary conditions, it is difficult to characterise the boundaries in an experiment. It is even more difficult to characterise them accurately. Since we do not need to prescribe the boundaries to perform the identification, we are not limited by the choice between using 
free boundary conditions (thus making the experimental setup complex) or accurately characterising the boundaries (thus making the experimental setup very complex). We can use any type of boundary condition.

Another experimental advantage of the simultaneous fitting is that the absolute magnitude of the $\boldsymbol{w}_{x}$ is not important in the bending stiffness identification - the measured modal slope $\boldsymbol{w}_{x}$ can be arbitrarily scaled without affecting the identified bending stiffness $\boldsymbol{E} \boldsymbol{I}$.

\section{Optimal number of harmonics $n_{h}$}

This section deals with the uncertainty of the shearography measurements and how they affect the choice of the parameters for the Fourier-series basis functions. In particular, the required number of harmonics $n_{h}$ of the Fourier series is investigated. The noise and fitting analysis is based on an article by Pintelon and Schoukens [30].

The shearography measurement of the first resonant mode of a flat aluminium beam is used for the noise and fitting analysis. The geometry of the flat beam is given in table 3. Free-free boundary conditions are approximated by hanging the vibrating beam at the nodal points. Additional support, to stop large back and forth oscillations, is provided by rubber pins placed behind the beam. This beam support is shown in figure 1 . In this set-up, the $x$ coordinate of the nodal points needs to be computed before-hand from the assumed bending stiffness. When vibrations at several modal frequencies are acquired, the nodal points differ for each frequency so the hanging locations need to be re-positioned. This makes the free-free boundary condition set-up less practical when we want to acquire multiple frequencies.

To obtain quantitative data from shearography, we need phase-unwrapping [31], which in turn requires filtering the shearography images. The filtering is performed by a median filter with a kernel size of $3 \times 3$ pixels. This is the minimal filtering after which the unwrapping is still successful. After the filtering, however, the neighbouring points cannot be considered statistically independent, which is a requirement for the statistical analysis we perform here. Therefore, each third column of a filtered shearography image is used, making the columns 


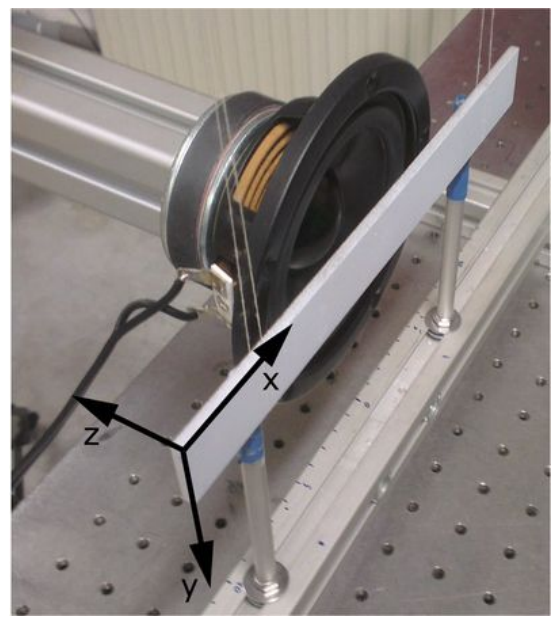

Figure 1: The experimental set-up with the free-free boundary condition. The beam is hung at the nodal points by thin threads. To avoid the beam undulations in the $z$-direction, the beam is additionally leaned against two rubber-coated pins.

independent and reducing the number of columns in the shearography images to $n=349$. The beam implies equations of one spatial variable, so the shearography images (2-dimensional fields) of the modal slope $w_{x}(x, y)$ are averaged over the width of the beam (the $y$ axis) to obtain a 1-dimensional modal slope vector $\boldsymbol{w}_{x}$.

To quantify the noise and its distribution, one shearography measurement is repeated a number of times. For a consistent estimate of the influence of measurement error in the parameter identification, the measurement should be repeated $n_{\text {meas }} \geq 6$ times (according to Schoukens et al. [32]). In this section, the measurement is repeated $n_{\text {meas }}=9$ times. The noise is not constant over the field-of-view in the shearography images, so the uncertainty is computed as a function of the $x$ coordinate.

All the possible sources of uncertainty need to be represented in the sample of $n_{\text {meas }}$ measurements. The laser illuminating the measurement creates a speckle pattern, which is instrumental for shearography imaging, but can also be seen as a source of measurement noise. Thus, for each measurement, the laser is slightly moved so that the speckle pattern changes between the measurements.

Two other sources of uncertainty stem from the discretization of a continuous signal in the CCD (the sensor of the shearography camera) and the unwrapping 


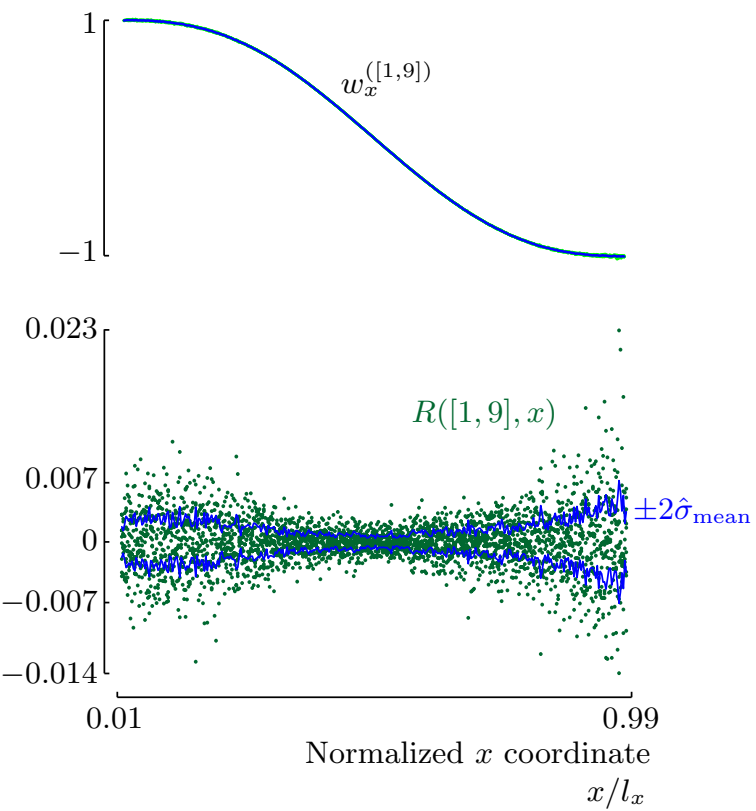

Figure 2: Measurement noise of the $n_{\text {meas }}=9$ repeated modal slope $\boldsymbol{w}_{x}$ is quantified. The measurements can be averaged and the error in a measurement is represented by the residuals $\boldsymbol{R}$. The standard deviation of the measurement is in the range $\hat{\sigma}_{x} \in$ $[0.0006,0.0102]$. For $n_{\text {meas }}=9$, this translates to $\pm 2 \hat{\sigma}_{\text {mean }}$ (which encompasses about $95 \%$ of residuals $\boldsymbol{R}$ ) of \pm 0.0004 at the minimum and \pm 0.0068 at the maximum.

algorithm. If the measurement is repeated at the constant modal slope amplitude, the uncertainty associated with discretization and unwrapping would not be properly accounted for. The modal slope amplitude is actually arbitrary in the bending stiffness identification - only the relative ratios are important. The measurements are thus performed at varying amplitudes and each measurement is normalized to the $w_{x} \in[-1,1]$ interval.

From the nine measurements of the modal slope $\boldsymbol{w}_{x}^{(i)}$, where superscript $(i)$ represents the $i$-th measurement, we can calculate the mean modal slope

$$
\hat{\boldsymbol{w}}_{x}=\frac{1}{n_{\text {meas }}} \sum_{i=1}^{n_{\text {meas }}} \boldsymbol{w}_{x, i}
$$

the $i$-th residual

$$
\boldsymbol{R}=\boldsymbol{w}_{x, i}-\hat{\boldsymbol{w}}_{x}
$$


the estimated variance

$$
\hat{\boldsymbol{\sigma}}^{2}=\frac{1}{n_{\text {meas }}-1} \sum_{i=1}^{n_{\text {meas }}} \boldsymbol{R}_{i}^{\top} \boldsymbol{R}_{i}
$$

and the estimated variance of the mean

$$
\boldsymbol{\sigma}_{\text {mean }}^{2}=\frac{\hat{\boldsymbol{\sigma}}^{2}}{n_{\text {meas }}}
$$

The optimal value for the number of harmonics $n_{h}$ to find the best fit according to equation (14) is now studied. As the starting value of $\hat{w}_{x}$ (see figure 2 , left side) is not equal to the end value (right side), the signal is step-like and performing the fit on it would not appropriately characterise it. Therefore, the fit is performed on a signal of double the physical length of the beam, $2 l_{x}$, as prescribed by the method for treating the step-like signals described by Gans and Nahman [33]. This method replaces the step-like signal by its analogue: a signal vector $s$ of length $n_{s}$ is replaced by the signal $s^{\prime}=\left[\begin{array}{c}s \\ -s+s(1)+s\left(n_{s}\right)\end{array}\right]$, of length $2 n_{s}$. The fundamental frequency of fitting $\Omega$ should be such that one period of the fitting function $2 \pi$ corresponds to $2 l_{x}$ on the $x$ coordinate. Thus $\Omega=\pi / l_{x}$. In practice this parameter is not fixed and can be tuned to slightly improve the fitting performance (reduction by about $10 \%$ usually gives best results). The optimal value of $\Omega$ cannot be given explicitly and can only be computed as a non-linear fitting parameter. To keep the equations reasonably simple, the default value $\Omega=\pi / l_{x}$ is kept for fitting and identification through this paper.

In case there are no systematic modelling errors, the expected value of the cost function can be computed, as described in [30] and [32]. In case there are modelling errors, such as if the number of harmonics used for fitting is too small, the actual cost function will be larger than its expected value. The expected value of the cost function depends on the number of fitting parameters, number of sampling points and on the noise in the measurement.

The diagonal weighting matrix $\boldsymbol{V}$ is used in equation (14) in the LLSQ fit, where each element on the diagonal is

$$
(\boldsymbol{V})_{i, i}=\frac{1}{\left(\boldsymbol{\sigma}_{\text {mean }}^{2}\right)_{i}}
$$


Table 1: For $n_{h} \geq 4$ the cost function $K_{\text {fit }}$ does not diminish significantly and the cost function $K_{\text {fit }}$ matches its expected value $\mathrm{E}\left\{K_{\text {fit }}\right\}$.

\begin{tabular}{rrc}
\hline$n_{h}$ & $K_{\text {fit }}$ & $\mathrm{E}\left\{K_{\text {fit }}\right\} \pm 2 \operatorname{std}\left\{K_{\text {fit }}\right\}$ \\
\hline 1 & 376400 & $231 \pm 20$ \\
2 & 5469 & $229 \pm 20$ \\
3 & 526 & $228 \pm 20$ \\
\hline 4 & 236 & $227 \pm 20$ \\
\hline 5 & 234 & $225 \pm 20$ \\
6 & 230 & $224 \pm 20$ \\
7 & 224 & $223 \pm 20$ \\
$\vdots$ & $\vdots$ & $\vdots$ \\
\hline
\end{tabular}

The expected value of the cost function $K_{\text {fit }}$ is then (according to Schoukens et al. [32])

$$
\mathrm{E}\left\{K_{\mathrm{fit}}\right\}=\frac{n-\left(2 n_{h}+1\right)}{2} \frac{n_{\text {meas }}-1}{n_{\text {meas }}-3}
$$

where $n=349$ is the number of measurement points on the curve and $2 n_{h}+1$ is the number of estimated parameters. Scaling of $\left(n_{\text {meas }}-1\right) /\left(n_{\text {meas }}-3\right)$ is to account for the fact that an estimated variance of the mean $\hat{\sigma}_{\text {mean }}^{2}$ is used, estimated from $n_{\text {meas }}$ measurements. The variance and the standard deviation of the cost function can be calculated as (from [32])

$$
\operatorname{var}\left\{K_{\mathrm{fit}}\right\}=\frac{n-\left(2 n_{h}+1\right)}{2} \frac{\left(n_{\text {meas }}-1\right)^{3}}{\left(n_{\text {meas }}-5\right)\left(n_{\text {meas }}-3\right)^{2}}
$$

and

$$
\operatorname{std}\left\{K_{\mathrm{fit}}\right\}=\sqrt{\operatorname{var}\left\{K_{\mathrm{fit}}\right\}}
$$

In case there are no modelling errors, the cost function is in the range

$$
K_{\text {fit }} \in\left[\mathrm{E}\left\{K_{\text {fit }}\right\} \pm 2 \operatorname{std}\left\{K_{\text {fit }}\right\}\right]
$$

with a $95 \%$ certainty.

Table 1 shows the value of the cost function $K_{\text {fit }}$ when fitting by $n_{h} \in$ $\{1, \ldots, 7\}$ complex harmonics and the expected value of the cost function. A fit by $n_{h}=4$ harmonics is thus sufficient to model the modal slope $\hat{w}_{x}$ with accuracy within the measurement uncertainty. 
In a next step, the optimal number of harmonics $n_{h}$ is investigated for the bending stiffness identification. In principle, the bending stiffness identification algorithm fits two variants of the modal slope, $\tilde{\boldsymbol{w}}_{x}$ and $\tilde{\tilde{\boldsymbol{w}}}_{x}$, to the measured modal slope $\boldsymbol{w}_{x}$. In this section, we have repeated measurements of the same modal slope, since we use the mean modal slope $\hat{\boldsymbol{w}}_{x}$ instead of single measurement $\boldsymbol{w}_{x}$. Likewise, we take advantage of the availability of the local weighting matrix $\boldsymbol{V}$. Equation (29) is used to perform the LLSQ fitting. The cost function defined in (28) is used. The expected value of the cost function $K_{\text {ID }}$ is

$$
\mathrm{E}\left\{K_{\mathrm{ID}}\right\}=\frac{2 n-\left(2 n_{h}+3\right)}{2} \frac{n_{\text {meas }}-1}{n_{\text {meas }}-3}
$$

where $n$ is the number of measurement points on the curve (the measurement range is taken twice into account in the identification process), $2 n_{h}+3$ is the number of estimated parameters $\left(2 n_{h}+1\right.$ coefficients $c$, the bending stiffness $E I$ and the integration constant from equation (18)). The variance (as in equation (37)) and the standard deviation (as in equation (38)) of the cost function cannot be calculated because the $2 n$ measurement values are not statistically independent - they are two copies of the same $n_{\text {meas }}$ measurements.

Using the resonalyser procedure (for details see Sol [34] and Lauwagie et al. [35]), the bending stiffness of the first tested beam is estimated to be $E I=9.5$ Nm. In this section, exceptionally, we will identify bending stiffness as if the beam is completely homogeneous, so the identification is performed in $n_{\mathrm{ID}}=1$ point.

Table 2 shows the minimized cost functions $K_{\mathrm{ID}}$, the expected cost function $\mathrm{E}\left\{K_{\mathrm{ID}}\right\}$ and the identified bending stiffness $E I$ for an increasing number of complex harmonics $n_{h}$. In the case of identification, a larger number of harmonics $n_{h}$ is needed than in the case of fitting. After $n_{h}=11$ the cost function does not diminish further, and the actual value of $E I$ is found. The identification of $E I$ converges slower (i.e. $K_{\mathrm{ID}}$ diminishes slower by increasing the number of harmonics) than the fitting (table 1 ). The identification at $n_{h} \geq 11$ is approximately as good as fitting at $n_{h} \geq 4$. In those cases the expected and the actual cost functions match.

In the case of bending stiffness identification, the LLSQ is doubly bound - both the function $\tilde{\boldsymbol{w}}_{x}$ and its fourth differential $\tilde{\tilde{\boldsymbol{w}}}_{x}$ must fit the measured $\hat{\boldsymbol{w}}_{x}$. The actual minimized cost function $K_{\mathrm{ID}}$ does not reach the expected value 
Table 2: From $n_{h} \geq 11$ the cost function $K_{\mathrm{ID}}$ converges to the minimal value and the bending stiffness $E I$ is properly identified.

\begin{tabular}{rrrr}
\hline$n_{h}$ & \multicolumn{1}{c}{$K_{\mathrm{ID}}$} & $E\left\{K_{\mathrm{ID}}\right\}$ & identified $E I$ \\
\hline 1 & 752800 & 462 & 46.3 \\
2 & 329623 & 461 & 40.4 \\
3 & 319651 & 459 & 37.5 \\
4 & 67316 & 458 & 18.8 \\
5 & 14451 & 457 & 18.4 \\
6 & 12004 & 455 & 18.7 \\
7 & 11980 & 454 & 18.7 \\
8 & 9450 & 453 & 14.6 \\
9 & 1343 & 451 & 9.72 \\
10 & 591 & 450 & 9.55 \\
\hline 11 & 524 & 449 & 9.54 \\
\hline 12 & 520 & 447 & 9.54 \\
13 & 519 & 446 & 9.54 \\
14 & 518 & 445 & 9.54 \\
$\vdots$ & $\vdots$ & $\vdots$ & $\vdots$ \\
\hline
\end{tabular}

$\mathrm{E}\left\{K_{\mathrm{ID}}\right\}$, though it comes close to it.

In this section, we show that the Fourier series is a very good basis function for the fitting. For the first resonant mode of a beam, only $n_{h}=4$ complex harmonics are sufficient to fit the measurement, within the precision allowed by the measurement noise. The bending stiffness identification procedure is based on simultaneous fitting of a Fourier series function and its fourth differential. In the case of identification, a higher number of coefficients is needed, $n_{h} \geq 11$.

\section{Identification from simulated data}

After defining the new identification algorithm and selecting the parameters, we can actually use it on test cases - that way we can assess the algorithm's accuracy, robustness and reliability. In this section we start from the simplest 
test case, a flat beam, towards more complex ones.

As shown in the previous sections, the input of the identification algorithm is the modal slope $\boldsymbol{w}_{x}$. We can either obtain the modal slope $\boldsymbol{w}$ from an experiment or generate it in a numerical simulation. Using simulations, in this case the finite-element ( $\mathrm{FE})$ method, has two major advantages: we know the exact bending stiffness in the model and the modal slope $\boldsymbol{w}_{x}$ is free of measurement noise and uncertainty. The FE model can be considered as a virtual experiment.

Five types of beams are tested. The first three, the flat, concave and convex beam, are both simulated and are available as physical samples. The fourth test beam has two sections with jumps in stiffness between them. Because of the difficulties involved in making a physical sample with accurately varying stiffness it is available only as a FE model. The fifth test beam is a flat beam with a weakened region (a region of reduced bending stiffness). It is both simulated and available as a physical sample, but the properties of the weakened region are slightly different in the simulation and in the physical sample. The last two beams are intended for testing how well the identification handles sharp discontinuities. The properties of all beams are summarized in the table 3 .

Table 3: Properties of the five types of aluminium beams

\begin{tabular}{lrrrrr}
\hline & Flat & Concave & Convex & $\begin{array}{r}\text { Sectioned } \\
\end{array}$ \\
\cline { 2 - 6 } & & & & $\begin{array}{r}\text { Locally } \\
\text { weakened }\end{array}$ \\
\hline Length $l_{x}$ in $\mathrm{mm}$ & 220.46 & 220.09 & 220.09 & 220.00 & 220.00 \\
Width $l_{y}$ in $\mathrm{mm}$ & 24.96 & 25.05 & 25.02 & 25.00 & 25.00 \\
Thickness $b$ in $\mathrm{mm}$ & 3.96 & {$[2.00,3.99]$} & {$[1.97,4.01]$} & 4.00 & {$[2.71,4.00]$} \\
Stiffness $E$ in $\mathrm{GPa}$ & 69.7 & 69.7 & 69.7 & $13.8,69.7$ & 69.7 \\
Available as: & & & & & $\checkmark$ \\
$\quad$ FEM & $\checkmark$ & $\checkmark$ & $\checkmark$ & $\checkmark$ & $\checkmark$ \\
$\quad$ Experiment & $\checkmark$ & $\checkmark$ & $\checkmark$ & & $\checkmark \mathrm{E}_{1} \perp \mathrm{E}_{2}$ \\
\hline
\end{tabular}

The algorithm can identify bending stiffness from modal slope $\boldsymbol{w}_{x}$ of any resonant mode. In the following examples the first five resonant modes are used from the finite-element model and the experiments. An advantage of this identification algorithm is that a combination of modal slopes $\boldsymbol{w}_{x}$ of different 
modes can be used, which makes the identification more robust. The improved robustness when using combined modal slopes $\boldsymbol{w}_{x}$ is discussed and demonstrated in section 4.2 .

We need a quantification parameter to measure how well the algorithm performs an identification, a goodness of identification. In the domain of curve fitting a common way to represent the goodness of fit is by the root-meansquare-deviation (RMSD) and we will adapt it for use here as well. For this particular problem it is defined as

$$
\operatorname{RMSD}=\sqrt{\operatorname{mean}\left(\left(\boldsymbol{E} \boldsymbol{I}-\boldsymbol{E} \boldsymbol{I}_{\mathrm{ID}}\right)^{\top}\left(\boldsymbol{E} \boldsymbol{I}-\boldsymbol{E} \boldsymbol{I}_{\mathrm{ID}}\right)\right)}
$$

where $\boldsymbol{E} \boldsymbol{I}$ is either the correct bending stiffness. The identification is commonly (and expected to be) inaccurate at the edges of the beam, so it is better to define the RMSD only in the region $x \in\left[0.1 l_{x}, 0.9 l_{x}\right]$.

To compare the results of identification for different beams between themselves, as RMSD is dependent on the actual local bending stiffness of the beam, the RMSD is normalized by the mean value of the correct (or estimated) bending stiffness $\boldsymbol{E} \boldsymbol{I}$. The resulting quantity is the coefficient of variation of the deviation

$$
\mathrm{CVD}=\frac{\mathrm{RMSD}}{\operatorname{mean}(\boldsymbol{E I})}
$$

and it is expressed as a percentage (of deviation from the mean bending stiffness).

The identification algorithm has some parameters which can be freely chosen. To keep the identification results fair, and to show that the algorithm is robust, all the identifications are run with the same parameters, unless noted otherwise. The identification is performed at $n_{\mathrm{ID}}=20$ points, with intermediate values interpolated by cubic splines. This is deemed a good compromise between fast identification (identification of a single mode takes about 2 to 3 seconds), ability to detect small deviations and robustness to noise. As we have seen in the previous section, $n_{h} \geq 11$ is needed for good identification. However, we use $n_{h}=12$ harmonics of the Fourier series for fitting, to account for the fact that we are using not just the first, but also higher vibrational modes. The initial guess for each identification is a constant field $E I=5 \mathrm{Nm}^{2}$. 


\subsection{Finite-element models}

The finite-element simulation is done in ABAQUS. Beams are modelled using a 2-dimensional mesh of ABAQUS S4R elements - each of which is a 4-node linear general-purpose shell element with reduced integration. S4R are mixed thin/thick shell elements, however, the thick shell formulation is forced. The mesh is of size $220 \times 20$ (each element is $1 \times 1.25 \mathrm{~mm}$ ), which is chosen to keep the individual elements approximately square and to have enough nodes in the $x$ direction to represent a smooth modal slope curve. An out-of-plane modal shape $w$ for each considered resonant frequency is the output of the simulation. From the 2-dimensional modal shape $w$ field, the 1-dimensional modal slope $\boldsymbol{w}_{x}$ curve is computed by differentiation in the $x$ direction (using central differences) and by averaging in the $y$ direction (the same averaging is done for the experimental data).

For vibrations, the physical characteristic which governs whether thin- or thick-beam behaviour can be expected is the wavelength of the modal shape (the distance between two peaks in the modal shape $w$ ). For higher modes the wavelength is shorter and as such the beam is approaching the thick-beam behaviour. As the identification algorithm is based on the thin-beam model, and thick plates are used for modelling, increasing discrepancies can be expected for the higher modes. Through this we can see how suitable the thin-beam implementation is for this problem.

The identification is performed for the modes $f_{1}$ to $f_{5}$ individually, and for all five modes combined. From figure 3 we can see the first mode having difficulties converging to the correct bending stiffness EI. For the rest of the modes, as well as when identifying the bending stiffness from the combined modes, the identification converges to the right values. This behaviour is seen also in the further examples and is likely because the lowest modes provides less information to the identification algorithm. However, all these identification results are actually very satisfactory - even the worst case has only CVD $=1.4 \%$. In general we can conclude that the identification algorithm can robustly perform the identification, in every vibration mode and by using combined modes. 


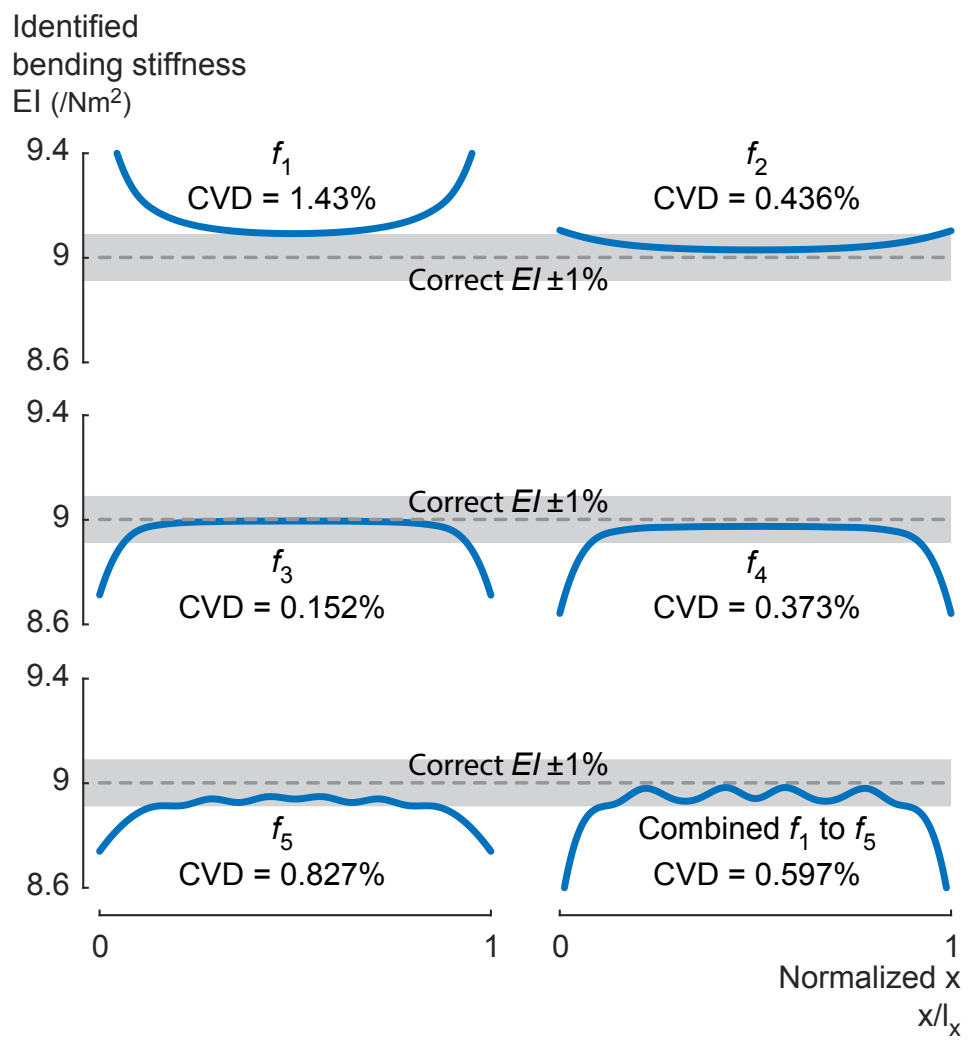

Figure 3: Identification from the finite-element flat beam model. Except for the first mode, the identification is within the $\pm 1 \%$ region of the correct bending stiffness value for $x / l_{x} \in[0.1,0.9]$.

\subsection{Robustness to noise}

An important feature of any identification method is its robustness to the measurement noise. If the identification method is overly sensitive to noise, it cannot be applied to real measurements. The identification method presented here is aimed at shearography measurements, which can be very noisy, thus a considerable robustness is needed. We show that the simultaneous Fourier-series identification method effectively deals with the measurement noise.

To accurately determine the robustness to noise we use data from the FE model, for which we know the exact bending stiffness and thus can exactly calculate the identification error. Normally distributed noise with standard deviation $\sigma_{\text {noise }}$ is added to the normalized modal slope slopes $\boldsymbol{w}_{x}$ (which are 


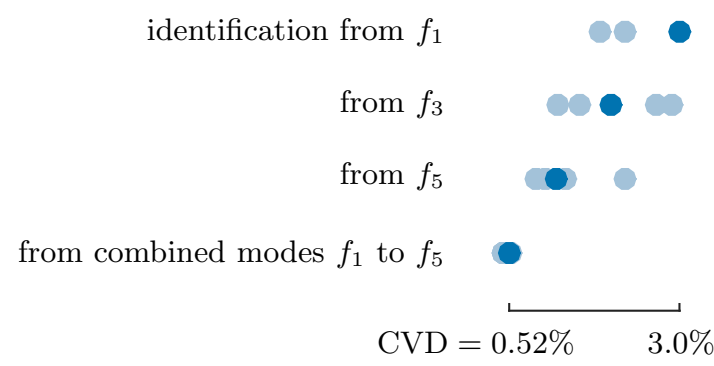

Figure 4: The higher the mode, the better robustness to noise - identification from modes $f_{3}$ and $f_{5}$ is considerably better than identification from mode $f_{1}$ for the same amount of noise. Identification becomes especially robust to noise when several modes are combined (here modes $f_{1}$ to $f_{5}$ ). The example is the flat beam with $\sigma_{\text {noise }}=10^{-2}$, which is on the upper limit of the expected amount of noise.

always in the range $[-1,1])$. In this section, the examples related to the noise robustness are focused on the flat beam, typically with noise of $\sigma_{\text {noise }}=10^{-2}$ added to the modes $f_{1}$ to $f_{5}$. In figure 2 we show that $\hat{\sigma}_{x} \in[0.0006,0.0102]$ so the noise that is used in the simulations is on the upper boundary of what can be expected in real measurements. Typically the identifications are performed from combined modes $f_{1}$ to $f_{5}$, without explicitly prescribing the boundary conditions. Since the added noise is by definition random, a slightly different result can be expected each time we run an identification. Each identification is performed five times, with random noise generated each time anew. For each identification, results of all five runs are shown in graphs, with the median value shown more prominently.

The same amount of noise is added to different modes to see how identification is affected by the choice of the resonant mode. As we see in figure 4 , the higher resonant modes are less affected by noise. Higher modes are denser in information, as the change of the modal slope $\boldsymbol{w}_{x}$ per unit length is larger. It can be said that the signal content the identification uses is higher. However, it is not always practical to use the higher modes as they are more difficult to excite in an experiment and the use of the thin-beam model becomes less appropriate for the higher modes.

Comparison of how a different amount of noise $\sigma_{\text {noise }}$ affects the accuracy of the identification is shown in figure 5 . Up to $\sigma_{\text {noise }}=10^{-2}$ the noise has 


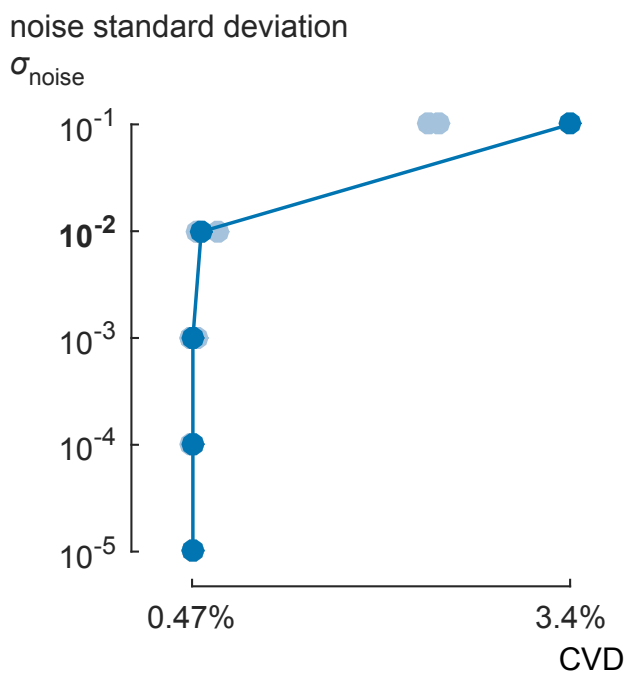

Figure 5: Up to the $\sigma_{\text {noise }}=10^{-2}$, which is on the upper level of the expected noise from shearography experiment, the identification from combined modes $f_{1}$ to $f_{5}$ is almost unaffected by noise. Even for the example with extreme noise $\sigma_{\text {noise }}=10^{-1}$, the identification error is acceptable.

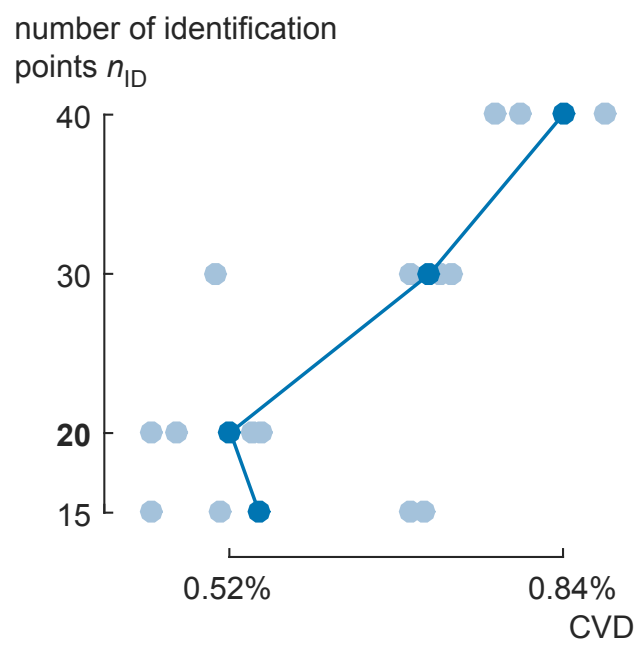

Figure 6: Robustness to noise is better, but not by much, if the number of identification control points is smaller. The points in this graphs are calculated for a flat beam, from numerical simulation of combined modes $f_{1}$ to $f_{5}$ with added noise of $\sigma_{\text {noise }}=10^{-2}$. 
number of harmonics $n_{h}$

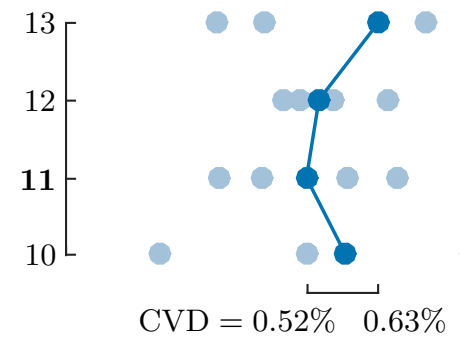

Figure 7: There is almost no change in the results when using different number of Fourier series harmonics $n_{h}$ for the bending stiffness identification. The points in this graphs are calculated for a flat beam, from numerical simulation of combined modes $f_{1}$ to $f_{5}$ with added noise of $\sigma_{\text {noise }}=10^{-2}$.

almost no effect on the results of the identification. Only at $\sigma_{\text {noise }}=10^{-1}$ is the identification significantly affected, though even in that case the median CVD is still acceptable, at $3.4 \%$. Bear in mind that $\sigma_{\text {noise }}=10^{-1}$ is an extreme example of noise, unlikely to be encountered in real shearography experiments.

When discussing the noise tolerance, an important parameter of the identification is the number of control points $n_{\mathrm{ID}}$, between which the bending stiffness is interpolated. The higher the number of control points $n_{\mathrm{ID}}$, the more locally the bending stiffness change is identified. However, smaller number of control points $n_{\mathrm{ID}}$ results in better robustness to noise. In figure 6 we see that an increase in the number of control points $n_{\mathrm{ID}}$ results in less noise robustness, but the effect is minor - the median CVD ranges from $0.52 \%$ for the best case to $0.84 \%$ for the worst case. The simultaneous Fourier-series identification is robust to noise, even when a large number of control points is used. The rest of the examples in this section use $n_{\mathrm{ID}}=20$ which gives the best noise tolerance and is fully capable to identify the bending stiffness change in all of the examples.

Another important parameter that needs to be selected for identification, that might affect the robustness to noise, is the number of Fourier-series harmonics $n_{h}$. In section 3 it is shown that the number of harmonics should be $n_{h} \geq 11$. Figure 7 further shows that the identification method is robust to the choice of the number of harmonics $n_{h}$. There is no statistically significant difference in choosing one over another. In all the other examples, the choice is set to $n_{h}=12$, as the higher number $n_{h}$ is computationally more expensive. 
In general, we can conclude that the simultaneous Fourier-series identification shows good robustness to noise, particularly when using higher modal shapes or, even better, when combining several distinct modal shapes.

\subsection{Identification from a subregion}

As shown already in section 2.3, the simultaneous Fourier-series identification method does not need information on the boundary conditions to identify the bending stiffness. When the modal slope $\boldsymbol{w}_{x}$ from only a subregion (a part of the beam of length $l_{\mathrm{ID}}$ ) is available, the influence of the rest of the beam can be replaced by some unknown boundary condition at the edges of the subregion. Since the algorithm does not need to have the boundary conditions prescribed anyhow, it is possible to identify the bending stiffness from only a subregion of the beam with no explicit information on the rest of the beam.

Figure 8 shows what happens when we try to identify the bending stiffness from a subregion (combined modes $f_{1}$ to $f_{5}$ are used) around the centre. If the subregion is large enough, the identification is successful, as seen from cases where identification length $l_{\mathrm{ID}} / l_{x}=\{40 \%, 60 \%, 80 \%\}$. If the subregion is too small, the identification fails to identify the correct bending stiffness.

Lower modes need larger identification length $l_{\mathrm{ID}}$ for a successful identification. In figure 9 , we see that for the mode $f_{1}$ almost the whole beam length is needed to successfully identify the bending stiffness. If we instead use the higher modes, a smaller subregion is enough - for the mode $f_{5}$ as little as $l_{\mathrm{ID}}=30 \% l_{x}$ is sufficient. Higher modes are more information-dense and bending stiffness identification can converge to the correct bending stiffness from information from such a small subregion. If we combine more modes, the bending stiffness identification needs larger identification length $l_{\mathrm{ID}}$ than the highest individual mode, but not much more, and combining modes has additional advantages, like better robustness to noise.

\subsection{Concave $\square$ and convex beam}

The concave beam and the convex beam have varying thicknesses. The concave beam is thin $(b=2.00 \mathrm{~mm})$ in the centre and thick $(b=3.99 \mathrm{~mm})$ at the edges. Conversely, the convex beam is thick $(b=4.01 \mathrm{~mm})$ at the center and thin 


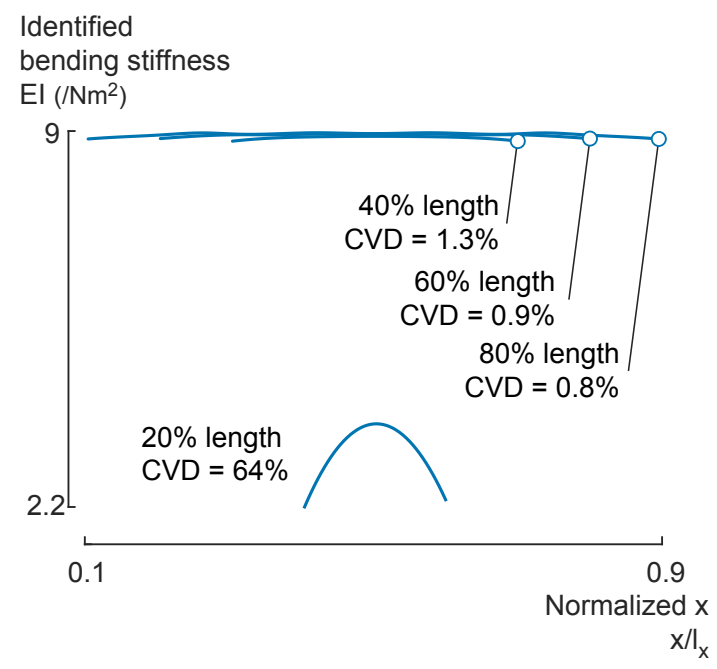

Figure 8: Several examples of identification from a subregion of a flat beam (combined data from modes $f_{1}$ to $f_{5}$ is used). When the subregion length is sufficient, the identification is successful, as in cases of identification from length $l_{\mathrm{ID}} / l_{x}=\{40 \%, 60 \%, 80 \%\}$. If the identification length is too small, such as in the example of $l_{\mathrm{ID}} / l_{x}=20 \%$, the identification converges to the wrong bending stiffness $E I$.

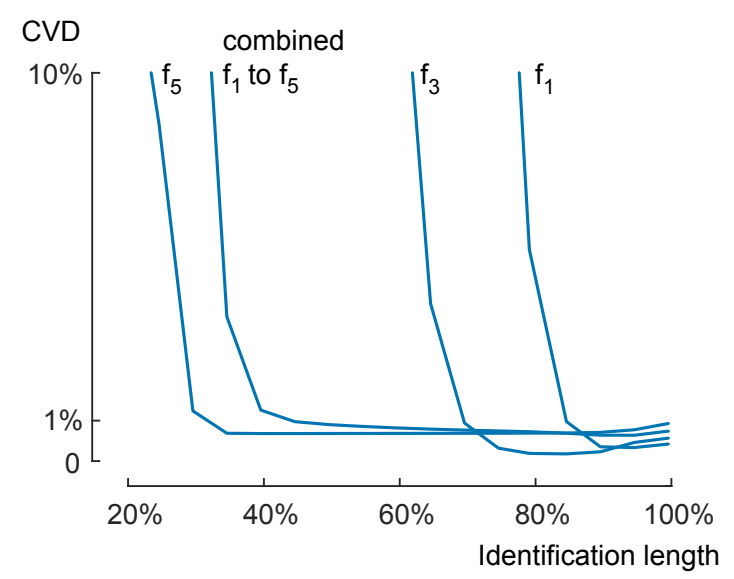

Figure 9: Depending on which resonant mode is used, identification is more or less robust to the choice of the identification subregion size $l_{\mathrm{ID}}$. We see that for mode $f_{1}$, the identification is only successful, i.e. the CVD $\leq 1 \%$, for $l_{\mathrm{ID}} / l_{x} \geq 85 \%$. For mode $f_{3}$ the identification is successful when $l_{\mathrm{ID}} / l_{x} \geq 85 \%$ and for mode $f_{5}$ identification can be successfully performed from as little as $30 \%$ length. When we combine modes (here shown combined modes $f_{1}$ to $f_{5}$ ) the identification needs a larger $l_{\mathrm{ID}}$ than when only using $f_{5}$ mode. 
$(b=1.97 \mathrm{~mm})$ at the edges. Other properties are similar to the flat beam, as shown in table 3 .

The bending stiffness computed from the FE model using the simultaneous Fourier-series identification is, in general, accurate, as seen in figures 10 and 11. For both the concave and convex beam, the identification from modes $f_{1}$ and $f_{2}$ converges to the wrong value. For those cases the CVD goes to above $600 \%$. This is in line, though more exaggerated, with what we have already seen in the case of the flat beam.

\subsection{Sectioned beam $E_{1} \perp E_{2}$}

We can use the example of the sectioned beam, to see if and what kind of artefacts a discontinuity in the bending stiffness $E I$ distribution causes in the identification. The sectioned beam is divided into two equally large sections, with abrupt and drastic change in bending stiffness between them (see figure 12).

When the bending stiffness identification is performed in the (default) number of identification points $n_{\mathrm{ID}}=20$, the identified bending stiffness shows oscillations on the left half of the beam. This is due to the Gibbs phenomenon (mentioned in section 2.3) - the Fourier series is unable to accurately fit the abrupt transitions such as here the discontinuity between the left and right side of the beam. A number of techniques have also been developed to deal with the Gibbs phenomenon, out of which the most successful ones are reviewed by Gottlieb and Shu [36]. In the future work, it should be researched which of them can be implemented in this identification framework. The Gibbs phenomenon can also be reduced if we increase the number of identification points. For example, by increasing the number of identification points to $n_{\mathrm{ID}}=100$ we see that although the unwanted oscillations of the bending stiffness are still present, they are less prominent and they affect a smaller region around the discontinuity. The downside of using a large number of identification points $n_{\mathrm{ID}}$ is that it makes the identification slightly more susceptible to the measurement noise. 


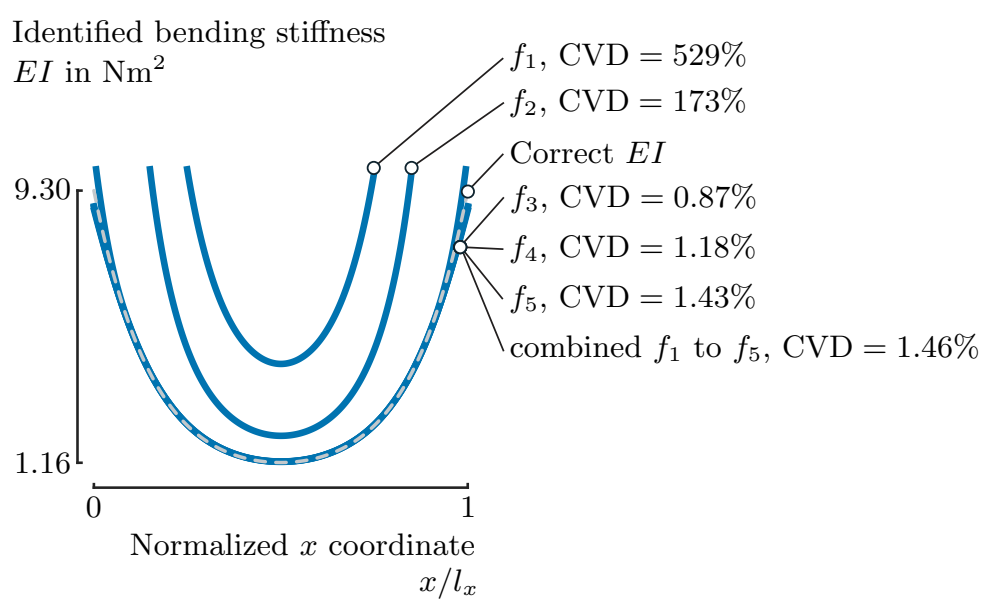

Figure 10: The identification of the bending stiffness of the FE model of the concave beam is accurate, except in cases when the identification is performed from the modes $f_{1}$ and $f_{2}$, for which the identification converges to wrong values.

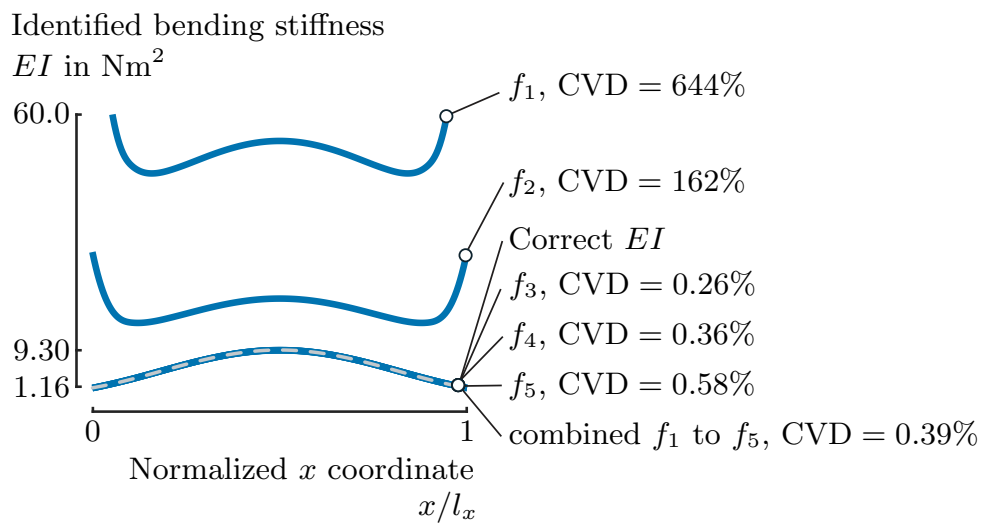

Figure 11: The identification of the bending stiffness of the FE model of the convex beam is accurate, except in cases when the identification is performed from the modes $f_{1}$ and $f_{2}$. 


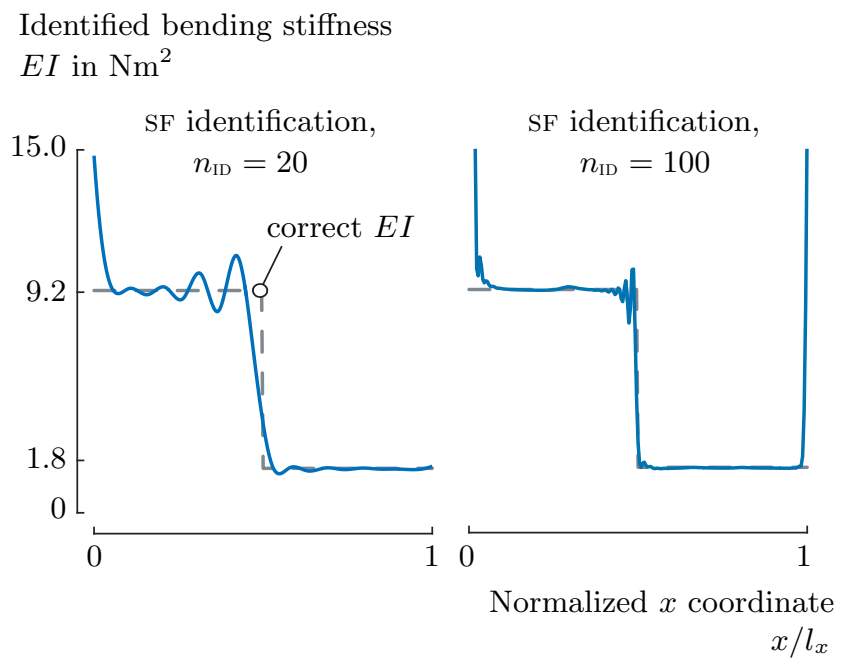

Figure 12: The abrupt transition in bending stiffness poses a problem for the identification methods. When using the simultaneous Fourier-series identification, there is an oscillation of bending stiffness near the transition point. When using a low number of identification points $(n=20)$ the situation is worse then when using a larger number $(n=100)$.

Identified bending stiffness

$E I$ in $\mathrm{Nm}^{2}$

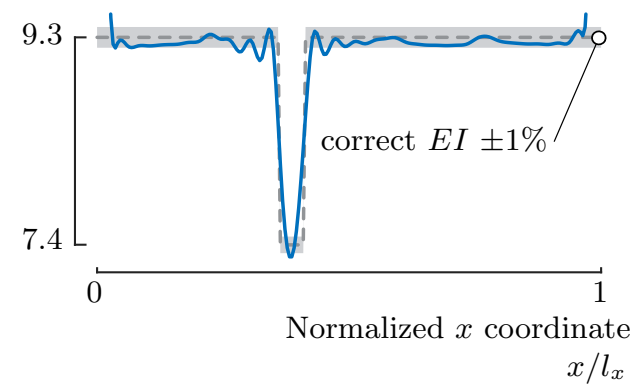

Figure 13: The bending stiffness of the weakened beam is identified accurately. 


\subsection{Locally weakened flat beam}

The weakened beam has a constant cross-section, the same as the flat beam. But, in the range $x / l_{x}=[0.36,0.41]$ the bending stiffness is reduced to $80 \%$ of the bending stiffness of the rest of the beam. This local weakening mimics a local damage to the beam.

The bending stiffness is identified from the combined five modes, in $n_{\mathrm{ID}}=50$ control points. The identified and the actual bending stiffness match very well, as seen in figure 13. In most of the beam's length the bending stiffness is identified to about $\pm 1 \%$ of the correct bending stiffness and the CVD $=1.6 \%$. We see that the simultaneous Fourier-series identification can successfully handle the abrupt changes in bending stiffness here.

\section{Identification from experiments}

For the beams which are available as physical samples, the modal slope $\boldsymbol{w}_{x}$ can of course be obtained from shearography experiments. This is the ultimate test of the identification method, but it has two drawbacks - we can only estimate the actual local bending stiffness EI distribution and the error of the identification is intertwined with the measurement uncertainty. This prevents a deeper quantitative analysis of the errors like in the previous section, but it still gives us a valuable qualitative insight into the behaviour of the identification method.

The measurement set-up is composed of the shearography camera, the beam support with clamping on one side and the excitation source. The shearography camera and the beam support are placed on a vibration-isolation table, to limit the vibration which deteriorates the shearography measurements. The shearography camera and the acquisition system is the isi-sys SE3 [37]. A loudspeaker behind the beam is used to excite the vibration at a resonant frequency. This way, a non-contact excitation is possible, and the mass-loading of the beam (as in the case when a shaker would be used) need not be taken into account. If there are significant energy losses due to the nature of the material (e.g. when using highly damped materials such as wood) or due to the losses at the boundary, the excitation sound can get very loud. The aluminium beams have a specularly-reflective surface. Since shearography can only image 


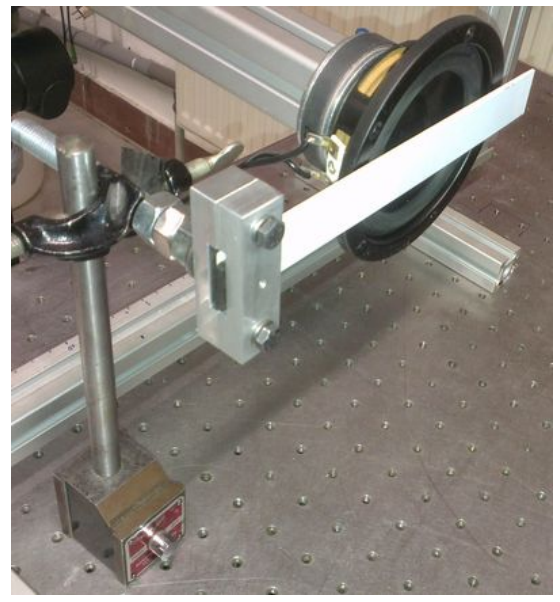

Figure 14: The experimental set-up with the clamped-free boundary condition is simple - the beam is clamped on the left side and multiple frequencies can be excited without changing the experimental setup.

diffusely-reflective surfaces, the beams are coated with a very thin layer of white powder.

The beams are manufactured from the same aluminium plate. The density $\rho=2676 \mathrm{~kg} / \mathrm{m}^{3}$ and Young's modulus $E=69.7 \mathrm{GPa}$ of the material are calculated from the flat beam, using the following procedure: the flat beam has a constant thickness $b=3.96 \pm 0.04 \mathrm{~mm}$ (measured in 23 points along the length); from the first resonant frequency $f_{1}=427.5 \mathrm{~Hz}$ (free-free boundary conditions), analytic relations for a flat beam calculate the average Young's modulus to $E=69.7 \mathrm{GPa}$; the density is calculated from the average measured thickness $b$, the length $l_{x}$, the width $l_{y}$ and the mass $m$. For a flat beam with average thickness $b$ and Young's modulus $E$, the corresponding bending stiffness is $E I=9.00 \pm 0.27 \mathrm{Nm}^{2}$. All the beams are prescribed with these material properties $(\rho$ and $E$ ) in the finite-element model in the previous section as well.

The beams are clamped at one side and free at the other, as shown in figure 14. The advantage of this setup, compared with the classical one shown in figure 1 , is that the locations of the nodal points are irrelevant for the measurement and thus we can easily excite multiple modes with no changes to the support. The clamped region of the beam cannot be imaged so no bending stiffness can be identified there. Clamping on both sides is not performed as the excitation 


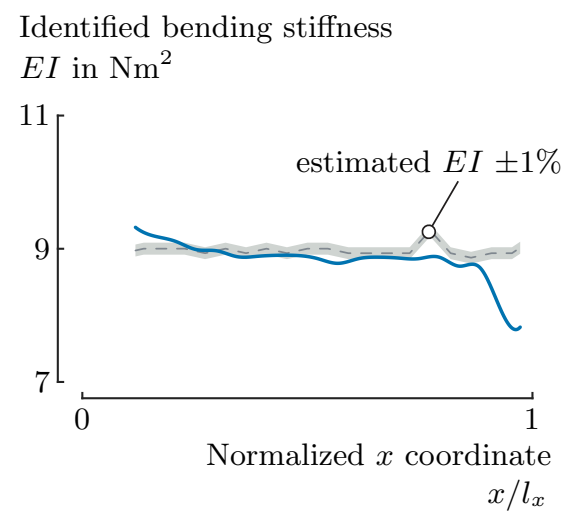

Figure 15: The identified bending stiffness $E I$ is similar to its assumed value when we perform the identification from the clamped-free beam and modes $f_{1}$ to $f_{5}$.

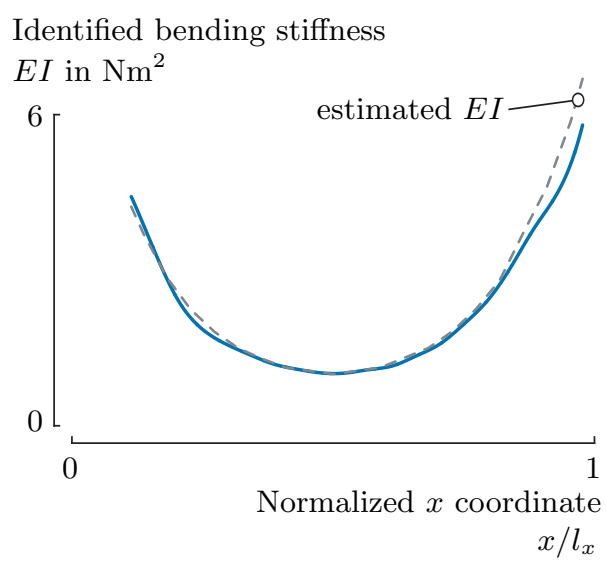

Figure 16: The results of the identification of the concave beam clamped on the left, from modes $f_{1}$ to $f_{5}$ matches very well with the estimated bending stiffness $E I$.

energy needed is too high to obtain good measurement for higher frequencies.

\subsection{Flat, concave and convex beam}

The identifications are performed from the combined first five modes. The identification result for the flat beam, in figure 15, is very close to the assumed value of the bending stiffness, with $\mathrm{CVD}=1.5 \%$. The Fourier-series identification performs a satisfactory identification from experimental measurements as well as the simulated data. The identification of the concave and the convex beam is also good, as seen in figures 16 and 17. 


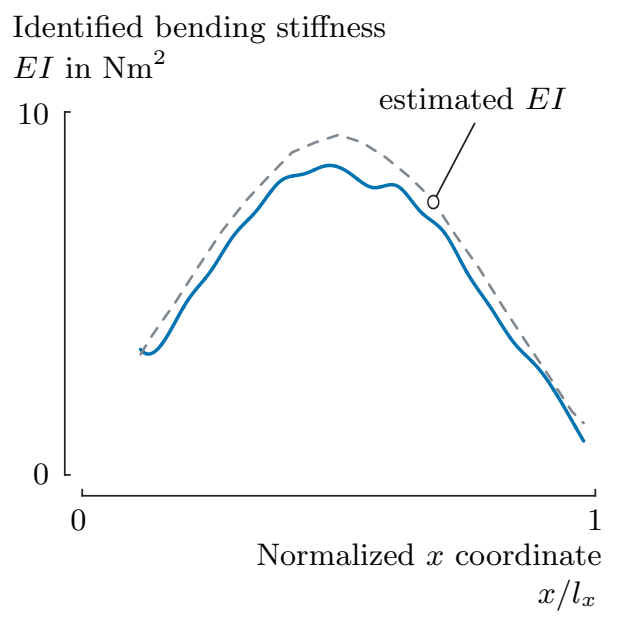

Figure 17: The bending stiffness identified from the convex beam clamped on left side, using combined modes modes $f_{1}$ to $f_{5}$.

\subsection{Locally weakened beam}

In the final experimental example, we simulate the damage by cutting a groove in a flat aluminium beam. This example also illustrates how well the identification performs when the decrease in bending stiffness is very localized. The groove is cut using a band saw, which ensures we obtain an even groove both in width and in depth. The groove can be progressively deepened, to track how the identification performs for different damage magnitudes. The groove is 1.45 $\mathrm{mm}$ wide, which is shown in figure 18. The identification is performed with the beam clamped on one side, like in the setup in figure 14 . We are exciting and measuring the first six resonant modes.

Since we are interested in the local bending stiffness, here we increase the number of identification points to $n_{\mathrm{ID}}=50$, compared to $n_{\mathrm{ID}}=20$ in the previous experiments. This allows for better local resolution in bending stiffness identification, though it will increase the identification uncertainty as well. With $n_{\mathrm{ID}}=50$, one identification point covers $l_{x} / n_{\mathrm{ID}}=4.4 \mathrm{~mm}$, which is three times the groove width $(1.45 \mathrm{~mm})$.

Normally, the bending stiffness EI for a rectangular cross-section of the beam is proportional to the cube of the local thickness $b$. A local reduction of thickness from $b_{\text {init }}$ to $b_{\mathrm{dmg}}$ thus causes a local bending stiffness reduction from $E I_{\text {init }}$ to $E I_{\mathrm{dmg}}=b_{\mathrm{dmg}}^{3} / b_{\text {init }}^{3} E I_{\text {init }}$. By cutting the groove we also remove 


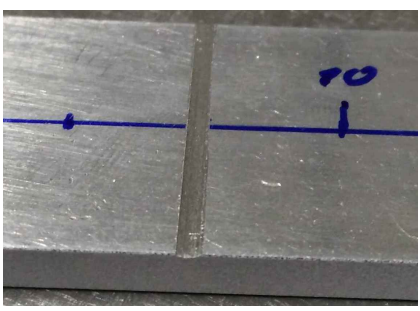

(a) Groove depth $0.28 \mathrm{~mm}$

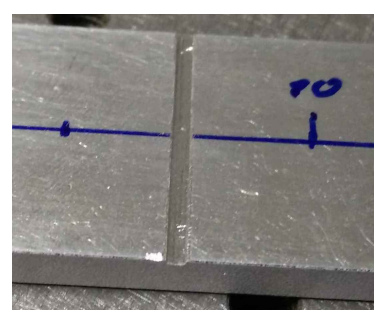

(b) Groove depth $0.52 \mathrm{~mm}$

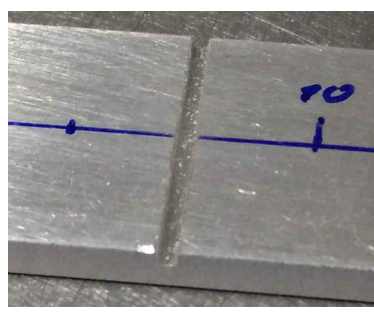

(c) Groove depth $1.29 \mathrm{~mm}$

Figure 18: To simulate damage in a flat aluminium beam, a thin groove of width $1.45 \mathrm{~mm}$ in thickness is cut. The groove is progressively made deeper, to track the simulated damage.

material. In the first approximation, the local mass does not affect the local bending stiffness so we do not have to explicitly prescribe this mass decrease in the identification. Also, the global mass change due to cutting the groove is negligible.

Figure 19 shows us the results of the identification, comparing the identification of the undamaged beam to the identifications with the groove depth of $0.28,0.52$ and $1.29 \mathrm{~mm}$. The identified bending stiffness outside of the groove before and after damage is very similar, though we can see that the relatively high $n_{\mathrm{ID}}$ results in fluctuation of the identified bending stiffness. The groove is clearly distinguishable in the identification for groove depths of 0.52 and 1.29 $\mathrm{mm}$. Even for the most shallow groove, there is a distinct difference in bending stiffness before and after damage. We can identify the local decrease of bending stiffness for the three groove depths as $91 \%, 79 \%$ and $43 \%$ of $E I_{\text {init }}$. However, due to the change in thickness the bending stiffness should be $80 \%, 66 \%$ and $31 \%$ of the original one. A reason for this mismatch might be that the groove width is much smaller than a single identification region.

\section{Conclusion and future work}

In this article, a novel local bending stiffness identification method, the socalled simultaneous Fourier-series identification, was developed. It is based on fitting the modal slope $\boldsymbol{w}_{x}$ to the two distinct variations of the Fourier-series, while enforcing the equations of the thin-beam theory. The method finds the 
Identified bending stiffness

$E I$ in $\mathrm{Nm}^{2}$

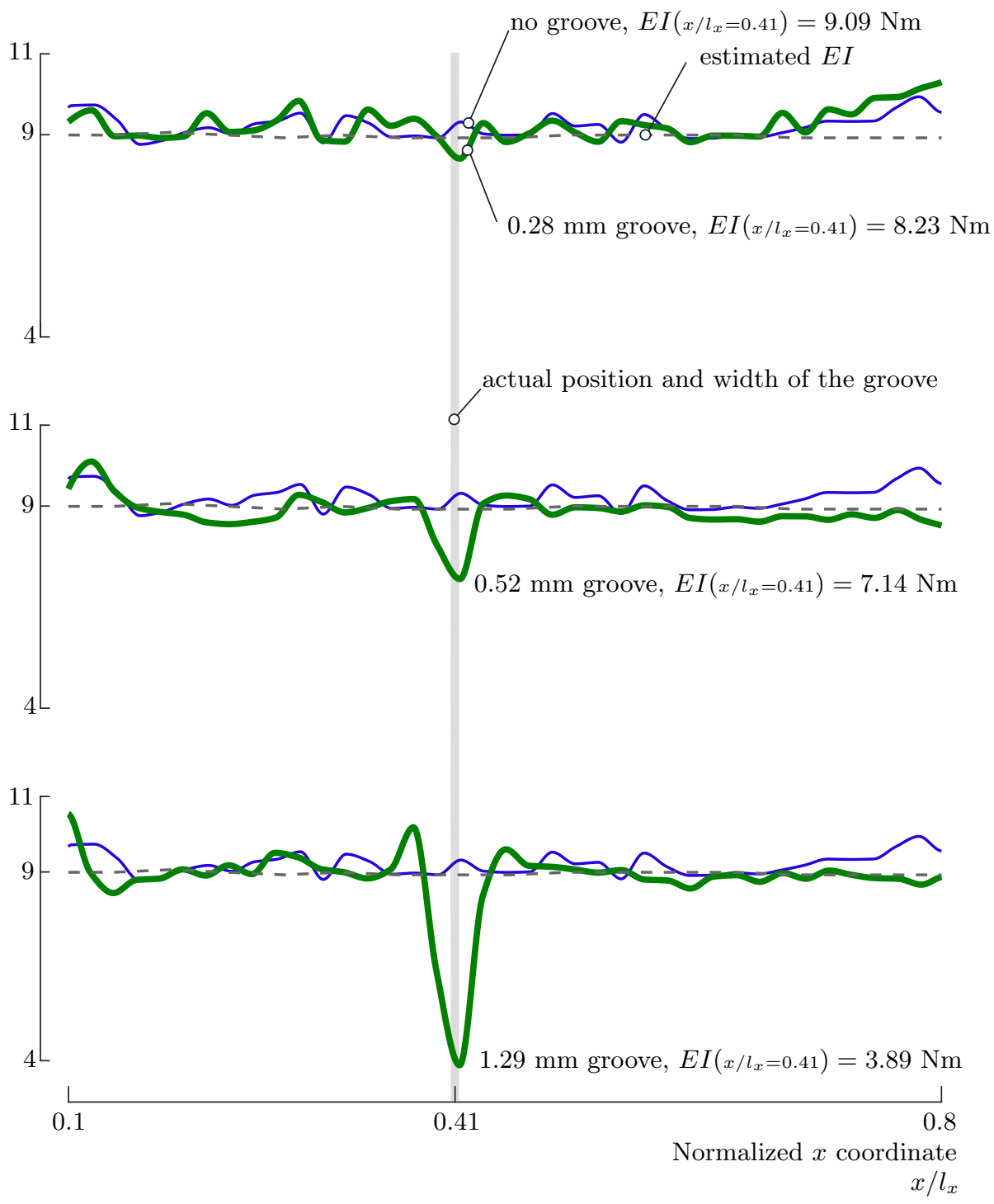

Figure 19: The identification is able to distinguish the change in the local bending stiffness for all three groove depths $(0.28,0.52$ and $1.29 \mathrm{~mm})$, though for the $0.28 \mathrm{~mm}$ groove the change in bending stiffness is within the uncertainty of the identification. The identified bending stiffness in the groove is not well matched to the bending stiffness which we would expect from the local thickness change, which is likely due to the fact that the groove width $(1.45 \mathrm{~mm})$ is much smaller than the length that one identification point covers $(4.4 \mathrm{~mm})$. 
bending stiffness distribution that fits the measured modal slope $\boldsymbol{w}_{x}$ from the shearography measurements.

Experimentally obtained modal slopes and modal slopes from finite-element (FE) model are used. Using the data from the FE model, the correct bending stiffness that should be obtained from the identification is known. This allows us to see how accurate the identification is; and to assess the robustness with respect to the measurement noise.

From the FE model we learn that the simultaneous Fourier-series identification is accurate - the results are in most cases at about $1 \%$ of the correct values. Simultaneous Fourier-series identification does not need to have boundary conditions explicitly prescribed (at least for modes higher than $f_{2}$ ). Practically, this means that the experiment can be performed with any boundary condition (it is not limited to the free boundary conditions only), which makes performing experiments simple and fast. Experiments are successfully performed with free-free and clamped-free boundary conditions. Another advantage of not explicitly prescribing the boundary conditions is that the bending stiffness can be identified from only a partially measured modal slope (from a subregion of the beam). In this article we show that the identification can be accurate from as little as $30 \%$ of the beam length.

We show that the simultaneous Fourier-series identification is robust to abrupt changes of the bending stiffness (same can be said for abrupt changes in thickness). The local bending stiffness for both the sectioned beam and the locally weakened beam can be accurately identified from the combined five modes. Fitting the sectioned beam by the Fourier series suffers from a misbehaviour near the discontinuities, the so-called Gibbs phenomenon. This can be reduced if we increase the number of identification points.

The effectiveness of the damage identification is experimentally verified by cutting a groove into a flat aluminium beam. By progressively deepening the groove we show that the identification can successfully track the progression of damage, even when the size of the damage is smaller than the size of a single identification region.

The simultaneous Fourier-series identification, as discussed in this article is used to identify the bending stiffness - similarly it can be used to identify the 
thickness of the beam. The non-linear optimization algorithm, which minimizes the cost function $K$ (equation (25)) by adjusting the local value of the $E I$ can perform the identical process by adjusting the local value of the thickness $b$ and keeping the Young's modulus $E$ constant. The rest of the thickness identification process is identical to the bending stiffness identification. This process was implemented on the same FE data and the thickness identification is as accurate as the bending stiffness identification. These results are omitted here for the sake of brevity.

Using weighted fitting (here used when calculating the optimal number of harmonics), makes capturing the modal slopes a more laborious process, since at least six measurements need to be done per mode that is captured. As the non-weighted fitting prove to be able to handle the noise very well, it was not necessary to make the modal slope process more complicated. However, if higher quality input is required, and especially if the process can be automated, it remains a viable option to increase the quality of the identification.

The thin-beam identification model will not be a good model to use when identifying bending stiffness of literally thick beams or beams made of sandwich material. Implementing the thick beam as the underlying model could further extend the usefulness of the identification method.

\section{References}

[1] M. Bonnet and A. Constantinescu, "Inverse problems in elasticity", Inverse problems, vol. 21, no. 2, R1, 2005.

[2] S. Avril, M. Bonnet, A.-S. Bretelle, M. Grédiac, F. Hild, P. Ienny, F. Latourte, D. Lemosse, S. Pagano, E. Pagnacco, and F. Pierron, "Overview of identification methods of mechanical parameters based on full-field measurements", Experimental Mechanics, vol. 48, no. 4, pp. 381-402, Jul. 2008.

[3] S. W. Doebling, C. R. Farrar, M. B. Prime, and D. W. Shevitz, "Damage identification and health monitoring of structural and mechanical systems from changes in their vibration characteristics: A literature review", Los Alamos National Lab., NM (United States), Tech. Rep., 1996. 
[4] C. Farrar and S. Doebling, "An overview of modal-based damage identification methods", Proceedings of DAMAS Conference, Sheffield, 1997.

[5] S. W. Doebling, C. R. Farrar, M. B. Prime, et al., "A summary review of vibration-based damage identification methods", Shock and Vibration Digest, vol. 30, no. 2, pp. 91-105, 1998.

[6] C. R. Farrar, T. A. Duffey, S. W. Doebling, and D. A. Nix, "A statistical pattern recognition paradigm for vibration-based structural health monitoring", Structural Health Monitoring, vol. 2000, pp. 764-773, 1999.

[7] C. Farrar, S. Doebling, and D. A. Nix, "Vibration-based structural damage identification", Philosophical Transactions of the Royal Society A: Mathematical, Physical and Engineering Sciences, vol. 359, no. 1778, pp. 131149, Jan. 2001.

[8] H. Sohn, C. R. Farrar, F. M. Hemez, D. D. Shunk, D. W. Stinemates, B. R. Nadler, and J. J. Czarnecki, "A review of structural health monitoring literature: 1996-2001", Los Alamos National Lab., NM (United States), Tech. Rep., 2004.

[9] A. Pandey, M. Biswas, and M. Samman, "Damage detection from changes in curvature mode shapes", Journal of Sound and Vibration, vol. 145, no. 2, pp. 321-332, Mar. 1991.

[10] J.-M. Ndambi, J. Vantomme, and K. Harri, "Damage assessment in reinforced concrete beams using eigenfrequencies and mode shape derivatives", Engineering Structures, vol. 24, no. 4, pp. 501-515, 2002.

[11] A. Huhtala and S. Bossuyt, "A Bayesian approach to vibration based structural health monitoring with experimental verification", Rakenteiden Mekaniikka (Journal of Structural Mechanics), vol. 44, no. 4, pp. 330-344, 2011.

[12] D. Dessi and G. Camerlengo, "Damage identification techniques via modal curvature analysis: Overview and comparison", Mechanical Systems and Signal Processing, vol. 52, pp. 181-205, 2015.

[13] J. Degrieck and W. Van Paepegem, "Fatigue damage modeling of fibrereinforced composite materials: Review", Applied Mechanics Reviews, vol. 54, no. 4, pp. 279-300, 2001. 
[14] R. Sevenois and W. Van Paepegem, "Fatigue damage modeling techniques for textile composites: Review and comparison with unidirectional composite modeling techniques", eng, Applied Mechanics Review, vol. 67, no. 2, B. Prorok, Ed., pp. 021401-1-021401-12, 2015.

[15] M. Kersemans, A. Martens, N. Lammens, K. Van Den Abeele, J. Degrieck, F. Zastavnik, L. Pyl, H. Sol, and W. Van Paepegem, "Identification of the elastic properties of isotropic and orthotropic thin-plate materials with the pulsed ultrasonic polar scan", Experimental Mechanics, vol. 54, no. 6, pp. 1121-1132, Mar. 2014.

[16] G. Kalogiannakis, B. Sarens, D. Van Hemelrijck, and C. Glorieux, "Shearographic detection of delaminations in sandwich structures: Investigation on various excitation modes", in Experimental Analysis of Nano and Engineering Materials and Structures, Springer, 2007, pp. 361-362.

[17] W. L. Lai, S. C. Kou, C. S. Poon, W. F. Tsang, S. P. Ng, and Y. Y. Hung, "Characterization of flaws embedded in externally bonded CFRP on concrete beams by infrared thermography and shearography", Journal of Nondestructive Evaluation, vol. 28, no. 1, pp. 27-35, Mar. 2009.

[18] G. De Angelis, M. Meo, D. Almond, S. Pickering, and S. Angioni, "A new technique to detect defect size and depth in composite structures using digital shearography and unconstrained optimization", NDTEEE International, vol. 45, no. 1, pp. 91-96, Jan. 2012.

[19] H. Lopes, F. Ferreira, J. Araújo dos Santos, and P. Moreno-García, "Localization of damage with speckle shearography and higher order spatial derivatives", Mechanical Systems and Signal Processing, vol. 49, no. 1-2, pp. 24-38, 2014.

[20] M. Mininni, S. Gabriele, H. Lopes, and J. Araújo dos Santos, "Damage identification in beams using speckle shearography and an optimal spatial sampling", Mechanical Systems and Signal Processing, pp. -, 2016.

[21] J. Maeck and G. De Roeck, "Dynamic bending and torsion stiffness derivation from modal curvatures and torsion rates", Journal of Sound and Vibration, vol. 225, no. 1, pp. 153-170, 1999. 
[22] Y. Goldfeld, "Identification of the stiffness distribution in statically indeterminate beams", Journal of Sound and Vibration, vol. 304, no. 3-5, pp. 918-931, Jul. 2007.

[23] Y. Goldfeld, "A direct identification procedure for assessment of stiffness distribution", Engineering Structures, vol. 31, no. 5, pp. 1068-1076, May 2009.

[24] Y. Goldfeld and D. Elias, "Using the exact element method and modal frequency changes to identify distributed damage in beams", Engineering Structures, vol. 51, pp. 60-72, Jun. 2013.

[25] T. T. Nguyen, J. M. Huntley, I. A. Ashcroft, P. D. Ruiz, and F. Pierron, "A Fourier-series-based virtual fields method for the identification of 2-D stiffness distributions", International Journal for Numerical Methods in Engineering, vol. 98, no. 12, pp. 917-936, 2014.

[26] T. Nguyen, J. Huntley, I. Ashcroft, P. Ruiz, and F. Pierron, "A Fourierseries-based virtual fields method for the identification of 2-D stiffness and traction distributions", Strain, vol. 50, no. 5, pp. 454-468, 2014.

[27] S. P. Timoshenko, History of strength of materials, With a brief account of the history of theory of elasticity and theory of structures. McGraw-Hill, 1953.

[28] S. S. Rao, Mechanical vibrations. Prentice Hall, Pearson, 2011.

[29] C. Moler, "Chapter 2 - Linear Equations", in Numerical computing with MATLAB, 2013.

[30] R. Pintelon and J. Schoukens, "An improved sine-wave fitting procedure for characterizing data acquisition channels", IEEE Transactions on Instrumentation and Measurement, vol. 45, no. 2, pp. 588-593, 1996.

[31] D. C. Ghiglia and M. D. Pritt, Two-Dimensional Phase Unwrapping: Theory, Algorithms, and Software. Wiley-Interscience, 1998, p. 512.

[32] J. Schoukens, R. Pintelon, and Y. Rolain, "Maximum likelihood estimation of errors-in-variables models using a sample covariance matrix obtained from small data sets", in Second international workshop Recent advances in total least squares techniques and errors -in-variables modeling, S. Van Huffel, Ed., 1996, pp. 59-68. 
[33] W. L. Gans and N. S. Nahman, "Countinuous and discrete Fourier transforms of steplike waveforms", IEEE Transactions on Instrumentation and Measurement, vol. IM-31, no. 2, pp. 97-101, 1982.

[34] H. Sol, "Identification of anisotropic plate rigidities by vibration analysis", $\mathrm{PhD}$ thesis, Vrije Universiteit Brussel, 1986.

[35] T. Lauwagie, H. Sol, G. Roebben, W. Heylen, and Y. Shi, "Validation of the resonalyser method: An inverse method for material identification", in Proceedings of ISMA2002, 2002, pp. 687-694.

[36] D. Gottlieb and C.-W. Shu, "On the gibbs phenomenon and its resolution", SIAM Review, vol. 39, no. 4, pp. 644-668, 1997.

[37] P. Mäckel, User manual and service instructions for SE3 shearography/ESPI, Dec. 2010. 